\title{
ČECH HOMOLOGY AND THE NOVIKOV CONJECTURES FOR K- AND L-THEORY
}

\author{
GUNNAR CARLSSON AND ERIK KJÆR PEDERSEN
}

\section{Introduction.}

In [5], we studied the assembly map in algebraic K- and L-theory, and showed that the assembly map splits for a class of groups $\Gamma$ with finite $B \Gamma$ for which $E \Gamma$ admits a metrizable, contractible, equivariant compactification such that the $\Gamma$-action is "small at infinity". This means that every compact subset of $E \Gamma$ when translated out near a point in the boundary becomes small i.e. for every $y \in \partial E \Gamma$ and for every neighborhood $U$ of $y$ in $\overline{E \Gamma}$, there is a neighborhood $V$ of $y$ so that $\gamma K \cap V \neq \emptyset$ implies $\gamma K \subset U$. The method used in [5] was to use continuously controlled $K$ - and $L$-theory.

Given a spectrum $S$ one may define homology with coefficients in the spectrum $S$ by the formula

$$
h_{*}\left(X, x_{0} ; S\right)=\pi_{*}(X \wedge S)
$$

for any finite pointed CW-complex $X$. If $X$ does not come exhibited with a basepoint we add a disjoint basepoint and get what is usually called unreduced homology by the formula $h_{*}(X ; S)=h_{*}\left(X_{+},+; S\right)=\pi_{*}\left(X_{+} \wedge S\right)$. When $X$ is not a CW-complex this does not give a good definition of the homology of $X$. The main theme of this paper is a Cech construction which gives a homotopy theoretically defined extension of such a functor to all compact Hausdorff spaces. Boris Goldfarb [10] has pointed out to us, that this construction is close to the constructions used by Edwards and Hastings $[8, \S 8.2]$, and can be seen as one possible solution to a problem posed by Edwards and Hastings [8, p. 251]. We refer the reader to [10] for further discussion of the history of this subject. We also construct natural transformations from various continuously controlled theories such as $K$-theory and $L$-theory to $\breve{C}$ ech theory. The theory satisfies the Steenrod axioms [13]. Hence using [14] the natural tranformation will be an isomorphism on the smaller category of compact metrizable spaces.

\footnotetext{
Research of the first author was supported in part by NSF DMS 8907771.A01.

Research of the second author was supported in part by NSF DMS 9104026.

Received December 15, 1995.
} 
As an application of this we show how this may be used to split assembly maps for various groups. We still need compactifications of $E \Gamma$ but we relax both the condition that the given compactification must necessarily be metrizable and the condition that the action be small at infinity.

The Čech homotopy type of a space $Z$ is the homotopy limit of the nerve of the finite coverings of $Z$. We say $Z$ is $\breve{C e c h}$ contractible if the $\breve{C e c h}$ homotopy type is contractible (in particular if $Z$ is contractible). If we are given a family $\mathscr{F}$ of open subsets of $Z$, the $\breve{C}$ ech homotopy type with respect to $\mathscr{F}$, is the homotopy limit of the nerve of finite coverings of $Z$, where we only use open sets from $\mathscr{F}$ in the finite coverings.

We prove the following theorems

Theorem A. Assume $\Gamma$ is a group with a finite $B \Gamma$ and that $E \Gamma$ has an equivariant compact Hausdorff compactification which is $\breve{C}$ ch contractible and such that the action is small at infinity then

a) If $R$ is any ring then the assembly map

$$
B \Gamma_{+} \wedge K^{-\infty}(R) \rightarrow K^{-\infty}(R \Gamma)
$$

is equivalent to an inclusion of a direct summand of spectra.

b) If $R$ is a ring with involution such that $K_{-i}(R)=0$ for $i$ sufficiently large then the assembly map

$$
B \Gamma_{+} \wedge \mathrm{L}^{-\infty}(\mathrm{R}) \rightarrow \mathrm{L}^{-\infty}(\mathrm{R} \Gamma)
$$

is equivalent to an inclusion of a direct summand of spectra.

We also have results about splitting assembly maps when the action is not small at infinity

Theorem B. Assume $\Gamma$ is a group with a finite $B \Gamma$ and that $E \Gamma$ has an equivariant compact Hausdorff compactification which is Čch contractible and such that there exists a family of coverings $\mathscr{F}$ of $\partial E \Gamma$ by sets which are boundedly saturated (see Definition 8.16), which is invariant under the group action and such that the Cech homotopy type defined by the family $\mathscr{F}$ is homotopy equivalent to the Čch homotopy type of $\partial X$. Then

a) If $R$ is any ring then the assembly map

$$
B \Gamma_{+} \wedge K^{-\infty}(R) \rightarrow K^{-\infty}(R \Gamma)
$$

is equivalent to an inclusion of a direct summand of spectra.

b) If $R$ is a ring with involution such that $K_{-i}(R)=0$ for $i$ sufficiently large then the assembly map

$$
B \Gamma_{+} \wedge \mathrm{L}^{-\infty}(\mathrm{R}) \rightarrow \mathrm{L}^{-\infty}(\mathrm{R} \Gamma)
$$

is equivalent to an inclusion of a direct summand of spectra. 
Boris Goldfarb in his Cornell thesis [10] has verified these conditions for various groups. Specifically he treats groups $\Gamma$ such that $\Gamma$ is the (torsion-free) fundamental group of a complete non-compact finite-volume Riemannian manifold with pinched negative sectional curvatures: $-b^{2} \leq K(M) \leq-a^{2}<0$. The $L$-theory assembly map was known to be split for this class of groups, but not the algebraic $K$-theory assembly map.

Throughout this paper we shall use the language of algebraic $K$-theory, the modifications needed to deal with $L$-theory are immediate using [5]. The results of this paper do work to split assembly maps in $A$-theory and topological $K$-theory as well, by using the excision results in [7] , [12] and [6]. For the readers convenience we state

Theorem C. Assume $\Gamma$ is a group as in Theorem A or B. Then

a) If $X$ is a space with $K_{-i}\left(\pi_{1}(X)\right)=0$ for $i$ sufficiently large, then the assembly map

$$
B \Gamma_{+} \wedge A_{-\infty}(X) \rightarrow A_{-\infty}(B \Gamma \times X)
$$

is equivalent to an inclusion of a direct summand of spectra.

b) If $C$ is a $C^{*}$-algebra then the assembly map

$$
B \Gamma_{+} \wedge K^{t o p}(C) \rightarrow K^{t o p}\left(C_{r}^{*} \Gamma\right)
$$

is equivalent to an inclusion of a direct summand of spectra.

We would like to thank the referee for numerous useful suggestions.

\section{Preliminaries.}

Throughout this paper, $s \mathscr{S}$ will denote the category of based simplicial sets and $\mathscr{K}$ will denote the full subcategory of Kan complexes. We will assume familiarity with the standard properties of homotopy inverse limits, also called homotopy limits, as presented in [3]. We shall conventionally only consider homotopy limits of Kan complexes, so if we ever encounter a homotopy limit in the category of simplicial sets, it is to be understood that we "Kan-ify" before taking the homotopy limit. Homotopy limits and colimits are extended to the category of spectra, by doing the constructions in each degree. If $C$ and $D$ are categories, $\Phi: D \rightarrow \mathscr{K}$ is a functor, and $f: C \rightarrow D$ is a functor, the induced map $\lim _{D} \Phi \rightarrow \lim _{C} \Phi \circ f$ will be referred to as pullback or restriction. Recall also that if $N: \Phi \rightarrow \Psi$ is a natural transformation of $\mathscr{K}$-valued functors on $D$, then $N$ induces a map $\lim _{D} \Phi \rightarrow \lim _{D} \Psi$. As mentioned above, we will only consider homotopy inverse limits of functors with values in $\mathscr{K}$. This means that the cosimplicial spaces used in defining 
the homotopy inverse limits are always fibrant, and all homotopy inverse limits will themselves be Kan complexes. A map of simplicial sets will be called a weak equivalence if its geometric realization is a homotopy equivalence. A map between Kan complexes is a weak equivalence if and only if it is a homotopy equivalence, i.e. there is a two-sided homotopy inverse map. We will refer to a weak equivalence between Kan complexes as an equivalence.

Similarly, if $\Phi: C \rightarrow s \mathscr{S}$ is any functor, we may construct the homotopy colimit of $\Phi$ over $C$, hocolim ${ }_{C} \Phi$. In this case we have pushforward maps corresponding to functors $f: C \rightarrow D$, and natural transformations again induce maps of homotopy colimits. If $\Phi, \Psi: C \rightarrow s \mathscr{S}$ are functors, and $N: \Phi \rightarrow \Psi$ is a natural transformation, we say $N$ is a weak equivalence if $N(c)$ is a weak equivalence for each object $c$ of $C$. Weak equivalences of $s \mathscr{S}$ valued functors induce weak equivalences on homotopy colimits, and weak equivalences of $\mathscr{K}$-valued functors induce weak equivalences of homotopy limits. We also recall from [4] that a homotopy natural transformation from a functor $\Phi$ to $\Psi$ is a sequence of functors $\Phi_{i}$ and $\Psi_{i}$, for $i=0,1, \ldots, k$, together with a family of natural transformations $\Phi_{i} \rightarrow \Psi_{i}$ and a family of natural equivalences $\Phi_{i} \rightarrow \Psi_{i-1}$ for $i>0$, where $\Phi_{0}=\Phi$ and $\Psi_{k}=\Psi$. A homotopy natural transformation induces a homotopy class of maps on homotopy colimits and homotopy limits.

If we have a diagram

$$
Y \stackrel{f}{\leftarrow} X \stackrel{g}{\rightarrow} Z
$$

in $s \mathscr{S}$, its homotopy pushout will be the double mapping cylinder $Y \coprod X \times I \coprod Z / \sim$, where $(x, 0) \sim f(x)$ and $(x, 1) \sim g(x)$. Note that this is the homotopy colimit of the diagram. We say a commutative diagram of simplicial sets

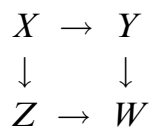

is homotopy co-Cartesian if the natural map $Y \cup_{X} Z \rightarrow W$ is a weak equivalence. Similarly, given a diagram

$$
Y \stackrel{s}{\rightarrow} W \stackrel{t}{\leftarrow} Z
$$

in $\mathscr{K}$ we define the homotopy pullback of the diagram as the subspace of $Y \times W^{I} \times Z$ of points $(y, \phi, z)$ so that $\phi(0)=y$ and $\phi(1)=Z$. $W^{I}$ here denotes the function complex of maps from the simplicial unit interval to $W$. This pullback is denoted by $Y \times_{W} Z$. We say a commutative diagram 


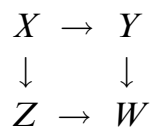

is homotopy Cartesian if the natural map $X \rightarrow Y \times_{W} Z$ is an equivalence.

Traditionally a homology theory was defined [1] to be a functor $h_{*}(X)$ from $\mathrm{CW}$-complexes to graded abelian groups satisfying the Eilenberg Steenrod axioms except for the dimension axiom. Using Brown's representability theorem [1] a homology theory has a representing spectrum $S$. This means that a homology theory can be written as $\pi_{*}\left(X_{+} \wedge S\right)$ for a suitable spectrum $S$ or $\pi_{*}(X \wedge S)$ for the corresponding reduced theory $h_{*}\left(X, x_{0} ; S\right)$ on pointed spaces. The functor $X \rightarrow X \wedge S$ from finite $\mathrm{CW}$-spaces to spectra is homotopy invariant and sends cofibrations of spaces to cofibrations of spectra and the one-point space to a contractible spectrum. The functor $X \rightarrow \Omega^{\infty} \Sigma^{\infty}(X \wedge S)$ from spaces to spaces sending $X$ to the 0 -th space of the infinite loop spectrum corresponding to $X \wedge S$ is homotopy invariant and sends cofibrations to fibrations. On the other hand if $f$ is a homotopy invariant functor sending cofibrations to fibrations and a point to a contractible space, it follows that $\pi_{*}(f(X))$ is a homology theory in the classical sense. It is easy to see that $\Omega f(\Sigma X))$ is homotopy equivalent to $f(X)$, so $f(X)$ is the 0 -th space of an infinite loop spectrum. This spectrum is the representing spectrum of the homology theory $\pi_{*}(f(X))$ as is shown in [19]. We may thus think of a homology theory in various equivalent ways. Since we shall need the results of [3] on homotopy limits and colimits it is practical to work simplicially rather than with spaces. We shall use the following definition:

Definition 2.1. A functor $T: s \mathscr{S} \rightarrow \mathscr{K}$ is said to be a homology theory if a) The induced map $T(X \times 0) \rightarrow T(X \times[0,1])$ is an equivalence for all simplicial sets $X$.

b) $T(*)$ is contractible.

c) For any homotopy co-Cartesian diagram

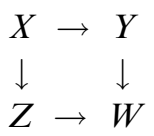

the induced diagram 


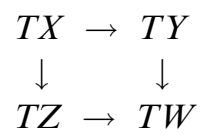

is homotopy Cartesian.

It follows that if we have an inclusion $i: X \hookrightarrow Y$, we obtain a sequence

$$
T X \rightarrow T Y \rightarrow T\left(Y \cup_{X} C X\right)
$$

which is a fibration "up to homotopy" in the sense that the natural map from $T X$ to the homotopy fiber of the map $T Y \rightarrow T\left(Y \cup_{X} C X\right)$ is an equivalence.

REMARK 2.2. Given a (simplicial) spectrum $\mathscr{S}$ we get a homology theory in this sense, by defining $T(X)$ to be the zero'th space of $\Omega^{\infty} S^{\infty}(X \wedge \mathscr{S}$ followed by a functor turning a simplicial set into a weakly equivalent Kan simplicial set.

We will also need standard information concerning the construction of spectra from category theoretic data. The following theorem covers what we will need. For more detail on the terminology in the statements, see [18].

THEOREM 2.3. There is a functor from the category of symmetric monoidal categories and lax symmetric monoidal functors to the category of spectra satisfying the following conditions.

1. If $f: \underline{C} \rightarrow \underline{D}$ is a lax symmetric monoidal functor and $N .(f)$, the induced map on the nerve, is a weak equivalence of simplicial sets, then $\operatorname{Spt}(f)$ is a weak equivalence of spectra.

2. For any symmetric monoidal category $\underline{C}$, let $\operatorname{Spt}_{0}(\underline{C})$ denote the zeroth space of the spectrum $\operatorname{Spt}(\underline{C})$. There is a natural map $N . \underline{C} \rightarrow \operatorname{Spt}_{0}(\underline{C})$, which induces an isomorphism

$$
\left(\pi_{0} N \cdot \underline{C}\right)^{-1} H_{*}(N . \underline{C}) \cong H_{*}\left(\operatorname{Spt}_{0}(\underline{C})\right)
$$

3. Let $f: \underline{C} \rightarrow \underline{D}$ be a unital symmetric monoidal functor between unital symmetric monoidal categories $\underline{C}$ and $\underline{D}$, and suppose $\pi_{0}(\underline{C})$ contains a cofinal submonoid $M$ so that $\pi_{0}(f)(M)$ is also a cofinal submonoid of $\pi_{0}(\underline{D})$. Suppose further that for every object $x \in \underline{D}$ lying in an equivalence class belonging to $\pi_{0}(f)(M), x \downarrow f($ or $f \downarrow x)$ has a weakly contractible nerve. Then $\pi_{i}((f))$ is an isomorphism for $i>0$.

4. If $\mu: \underline{A} \times \underline{B} \rightarrow \underline{C}$ is a symmetric monoidal pairing, then there is an induced pairing of spectra

$$
\operatorname{Spt}(\mu): \operatorname{Spt}(\underline{A}) \wedge \operatorname{Spt}(\underline{B}) \rightarrow \operatorname{Spt}(\underline{C})
$$

so that the composite 


$$
N . \underline{A} \times N . \underline{B} \rightarrow \operatorname{Spt}_{0}(\underline{A}) \wedge \operatorname{Spt}_{0}(\underline{B}) \rightarrow(\operatorname{Spt}(\underline{A}) \wedge \operatorname{Spt}(\underline{B}))_{0} \rightarrow \operatorname{Spt}_{0}(\underline{C})
$$

is equal to the composite

$$
N \cdot \underline{A} \times N \cdot \underline{B} \stackrel{N \cdot \mu}{\longrightarrow} N \cdot \underline{C} \rightarrow \operatorname{Spt}_{0}(\underline{C})
$$

Proof. The first 2 points are [18, Lemma 2.3] and [18, Condition 2.2], and 3. and 4. are proved in [4, Theorem I.6].

We also recall the notation $h\left(X, x_{0}, \mathscr{S}\right.$ for the homology of the based space $\left(X, x_{0}\right)$ "with coefficients in the spectrum $\mathscr{S}$ ", or the smash product of the space $X$ with the spectrum $\mathscr{S}$.

We will be using homotopy inverse limits over certain categories of coverings of topological spaces in our definition of Čech homology. These categories have certain properties which make them convenient to work with, and we discuss these now. Recall from [9] that a category $C$ is said to be left filtering if (i.) for every pair of objects $c, c^{\prime}$ in $C$, there exists an object $c^{\prime \prime}$ together with maps $c \longleftarrow c^{\prime \prime} \rightarrow c^{\prime}$, and (ii.) for every pair of maps $f, g: c^{\prime} \rightarrow c$, there exists a morphism $h: c^{\prime \prime} \rightarrow c^{\prime}$ so that $f \circ h$ and $g \circ h$ are equal. If the category $C$ happens to be a partially ordered set, i.e. there is at most one morphism between any pair of objects, then this reduces to the requirement that for any pair of objects $c, c^{\prime}$ of $C$, there is a $c^{\prime \prime}$ so that there are morphisms $c^{\prime \prime} \rightarrow c$ and $c^{\prime \prime} \rightarrow c^{\prime}$. We will adopt the convention that a partially ordered set in the usual sense is made into a category by declaring that there is a morphism from $x$ to $y$ if and only if $x \geq y$. If this category is left filtering we shall say the partially ordered set is left directed. Note that it follows as in [16] that the nerve of any left filtering category is weakly contractible, and hence the nerve of any left directed partially ordered set is weakly contractible.

Proposition 2.4. Let $C$ and $D$ be two left directed partially ordered sets, and suppose we have an order preserving map $f: C \rightarrow D$. Further, suppose that $\phi$ is a functor from $D$ to the category of Kan simplicial sets, and that for every element $z \in D$ there is an element $x \in C$ so that $f(x) \geq z$. Then the pullback map

$$
\underset{D}{\operatorname{holim}} \phi \rightarrow \underset{C}{\operatorname{holim}} \phi \circ f
$$

is a weak equivalence.

Proof. From [3], it will suffice to show that the category $f \downarrow z$ has contractible nerve for each $z \in D$. But it is clear that each category $f \downarrow z$ is itself a left directed partially ordered set, and the hypothesis of the proposition shows that it is non-empty. Therefore, its nerve is contractible.

We also have the following standard fact(see [3]). 
Proposition 2.5. Let $C$ be a category, and suppose that we have functors $F, G, H$ and $K$ from $C$ to the category of based Kan complexes, and that we have a commutative diagram of natural transformations as follows.

$$
\begin{array}{ll}
F & \rightarrow \\
\downarrow & \\
H & \rightarrow
\end{array}
$$

Suppose further that for each $c \in C$, the diagram is homotopy Cartesian, i.e. that the natural map from $F(c)$ to the homotopy pullback of the diagram $H(c) \rightarrow K(c) \longleftarrow G(c)$ is an equivalence. Then the diagram

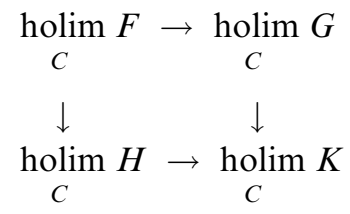

is also homotopy Cartesian.

We will also need some conditions which assure that a natural transformation between functors from a left directed partially ordered set to the category of Kan complexes induces a weak equivalence on homotopy inverse limits.

Proposition 2.6. Let $C$ denote a left partially ordered set, and suppose that we are given a natural transformation $\eta: F \rightarrow G$ of functors from $C$ to $\mathscr{K}$. Suppose further that for every $c \in C$, there is a $c^{\prime} \geq c$ so that $\eta\left(c^{\prime}\right)$ is an equivalence. Then the map holim ${ }_{C} F \rightarrow$ holim ${ }_{C} G$ induced by $\eta$ is an equivalence.

Proof. This is a straightforward consequence of [3].

We must also understand the behavior of restriction maps on inverse limits. Preparing for this we state

Lemma 2.7. Let $f: E \rightarrow C$ be an order preserving map of partially ordered sets, and $T: E \rightarrow$

Lemma $\mathscr{K}$ a functor. For every $y \in C$ let $E_{y}$ be the full subcategory of $E$ consisting of $y^{\prime}$ so that $y \geq f\left(y^{\prime}\right)$ then

$$
\underset{E}{\operatorname{holim}} T \simeq \underset{C}{\operatorname{holim}} \operatorname{holim}_{E} T \mid E_{y}
$$

Proof. Let $Z$ denote the partially ordered set consisting of pairs $(c, e)$ with $c \geq f(e)$. Then the iterated homotopy limit may be identified with the homotopy limit over $Z$ of the functor $T^{\prime}: Z \rightarrow \mathscr{K}$ sending $(c, e)$ to $T(e)$. 
Moreover there is a forgetful functor $i: Z \rightarrow E$ sending $(c, e)$ to $e$, and $T^{\prime}=T \circ i$. It is readily checked that $i$ satisfied the hypothesis of [3, Theorem XI.9.2]. Hence $i$ induces an equivalence on homotopy inverse limits.

Lemma 2.8. Let $C \subseteq D$ be an inclusion of left directed partially ordered sets. Let $F$ be a functor from $D$ to the category of Kan complexes. Suppose that for every $x \in D$, there exists $x^{\prime} \in D$ and $a y^{\prime} \in C$, with $x^{\prime} \geq x$ and $x^{\prime} \geq y^{\prime}$ and with $F\left(x^{\prime}\right) \rightarrow F\left(y^{\prime}\right)$ a weak equivalence. Then the restriction map $\operatorname{holim}_{D} F \rightarrow \operatorname{holim}_{C} F$ is a weak equivalence.

Proof. Let $E$ be the partially ordered set whose objects are pairs $(x, y)$, with $x \in D, y \in C$, and $x \geq y$. We have functors $r: E \rightarrow D$ and $i: C \rightarrow E$, with $r(x, y)=x$ and $i(y)=(y, y)$. Note that $r \circ i$ is equal to the inclusion $C \hookrightarrow D$. Let $\hat{E} \subseteq E$ be the full subcategory on all $(x, y)$ so that $F(x \geq y)$ is a weak equivalence. Of course, $i(C) \subseteq \hat{E}$. The hypothesis shows that the restriction of $r$ to $\hat{E}$ satisfies the hypotheses of 4, so the natural map

$$
\underset{D}{\operatorname{holim}} F \rightarrow \underset{\hat{E}}{\operatorname{holim}} F \circ r
$$

is an equivalence. It now suffices to show that the restriction map

$$
\underset{\hat{E}}{\operatorname{holim}} F \circ r \rightarrow \underset{C}{\operatorname{holim}} F \circ r \circ i
$$

is an equivalence. Let $T$ denote the functor from $\hat{E}$ to Kan complexes given by $T(x, y)=F(y)$. There is an evident natural equivalence of functors on $\hat{E}$ from $F \circ r$ to $T$, given by $F(x \geq y)$ so when restricted to $i(C)$ it gives the identity equivalence. Hence

$$
\underset{\hat{E}}{\operatorname{holim}} F \circ r \simeq \underset{\hat{E}}{\operatorname{holim}} T
$$

Note that $T \circ C=F \circ r \circ i$. Consequently, it suffices to show that the restriction $\operatorname{map} \operatorname{holim}_{\hat{E}} T \rightarrow \operatorname{holim}_{C} T \circ i$ is a weak equivalence. To prove this, given any $y \in C$, we let $\hat{E}_{y}$ denote the full subcategory on those $\left(x, y^{\prime}\right)$ for which $y \geq y^{\prime}$. We use Lemma 2.7 to express holim $\hat{E}_{\hat{E}} T$ as an iterated homotopy limit

$$
\underset{\hat{E}}{\operatorname{holim}} T \simeq \underset{y \in C}{\operatorname{holim}} \hat{E}_{y} T \mid \hat{E}_{y}
$$

it is easy to see that the diagram

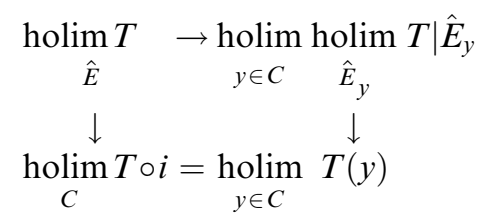


is commutative. The right hand vertical map is given by restriction along the inclusion $\{(y, y)\} \subseteq \hat{E}_{y}$. In view of 2.6, it thus suffices to check that $\operatorname{holim}_{\hat{E}_{y}} T \mid \hat{E}_{y} \rightarrow T(y)$ is an equivalence. Now, by 2.4 , it is clear that if $E_{y}^{*} \subseteq E_{y}$ is the subset on objects of the form $(x, y)$, then the restriction functor $\operatorname{holim}_{\hat{E}_{y}} T \rightarrow \operatorname{holim}_{E_{y}^{*}} T$ is an equivalence. Consequently, it suffices to check that the map holim $E_{v}^{*} T \rightarrow T(y)$ is an equivalence. But $T$ is constant on $E_{y}^{*}$ with value $T(y)$, so the homotopy limit over $E_{y}^{*}$ can be identified with the function complex $F\left(N . E_{y}^{*}, T(y)\right)$, and the restriction map is simply restriction along the inclusion of nerves of the one object category $(y, y)$ into $N$. $E_{y}^{*}$. But $N . E_{y}^{*}$ is weakly contractible since it has a final object $(y, y)$.

\section{Lemmas on Coverings.}

By a covering of a topological space $X$, we mean a parameterized family $\mathscr{U}=\left\{U_{\alpha}\right\}_{\alpha \in A}$ of open subsets of $X$, where $A$ is a set, so that $X=\bigcup_{\alpha \in A} U_{\alpha}$. A map of coverings from $\left\{U_{\alpha}\right\}_{\alpha \in A}$ to $\left\{V_{\beta}\right\}_{\beta \in B}$ is a set map $f: A \rightarrow B$ so that $U_{\alpha} \subseteq V_{f(\alpha)}$ for all $\alpha \in A$.

By a simplicial complex $\Sigma$ we mean, as usual, a vertex set $V_{\Sigma}$ and a family of finite subsets $\mathscr{P}_{\Sigma}$, so that if $U \in \mathscr{P}_{\Sigma}$ and $U^{\prime} \subseteq U$ then $U^{\prime} \in \mathscr{P}_{\Sigma}$. Simplicial maps are defined in the usual way. Two simplicial maps $f, g: \Sigma_{1} \rightarrow \Sigma_{2}$ are said to be $s$-homotopic if for every $U \in \Sigma_{1}, f(U) \cup g(U) \in \Sigma_{2}$. The join of two simplicial complexes $\Sigma$ and $\mathscr{T}, \Sigma * \mathscr{T}$, has $V_{\Sigma} \amalg V T$ as its vertex set, and a subset of $V_{\Sigma * \mathscr{T}}$ is in $\mathscr{P}_{\Sigma * \mathscr{T}}$ if and only if it is the union of an element of $V_{\Sigma}$ with an element of $V_{\mathscr{T}}$.

For any set $X$, let $\mathscr{F}(X)$ denote the partially ordered set of nonempty finite subsets of $X$ and inclusions. As usual we may view a $\mathscr{F}(X)$ as a category , which by our conventions is the opposite category of the category of nonempty subsets and inclusions. The functor $X \rightarrow N . \mathscr{F}(X)$ gives a covariant functor from $\underline{\text { Sets }}$ to $s \mathscr{S}$. Given a simplicial complex $\Sigma$, let the realization of $\Sigma, R . \Sigma$, be the nerve of the full subcategory of $\mathscr{F}\left(V_{\Sigma}\right)$ with objects the subsets belonging to $\mathscr{P}_{\Sigma}$. This is the simplicial version of the barycentric subdivision of the usual realization. The functor $R$. preserves pushouts and carries $s$-homotopic maps to simplicially homotopic maps.

For any covering $\mathscr{U}=\left\{U_{\alpha}\right\}_{\alpha \in A}$ of a topological space, let $\Sigma_{\mathscr{U}}$ be the simplicial complex whose vertex set is $A$, and where $\left\{\alpha_{1}, \ldots, \alpha_{k}\right\}$ is a simplex of $\Sigma_{\mathscr{U}}$ if and only if $U_{\alpha_{1}} \cap \ldots \cap U_{\alpha_{k}} \neq \emptyset$. By the nerve of the covering, $N$. $\mathscr{U}$, we will mean $R$. $\Sigma_{\mathscr{U}}$.

Lemma 3.1. If $\mathscr{U}$ and $\mathscr{V}$ are any coverings of $X$, and $f, g: \mathscr{U} \rightarrow \mathscr{V}$ are any maps of coverings, then $N . f$ and $N . g$ are simplicially homotopic.

Proof. This result follows directly from the above discussion of $s$-homo- 
topies, since if $f$ and $g$ are both maps of coverings from $\mathscr{U}$ to $\mathscr{V}$, and $\left\{\alpha_{1}, \ldots, \alpha_{k}\right\} \in \Sigma_{\mathscr{U}}$, then

$$
\left\{f\left(\alpha_{1}\right), \ldots, f\left(\alpha_{k}\right), g\left(\alpha_{1}\right), \ldots, g\left(\alpha_{k}\right)\right\} \in \Sigma_{\mathscr{V}}
$$

since

$$
U_{\alpha_{1}} \cap \ldots \cap U_{\alpha_{k}} \subseteq U_{f\left(\alpha_{1}\right)} \cap \ldots \cap U_{f\left(\alpha_{k}\right)} \cap U_{g\left(\alpha_{1}\right)} \cap \ldots \cap U_{g\left(\alpha_{k}\right)}
$$

Thus, $f$ and $g$ are $s$-homotopic.

Corollary 3.2. Let $f: \mathscr{U} \rightarrow \mathscr{V}$ be any map of coverings. Suppose further that there is a map of coverings from $\mathscr{V}$ to $\mathscr{U}$. Then $N$.f is a weak equivalence.

We will also define certain other simplicial sets associated to $\mathscr{U}$. Suppose $B \subseteq A$.

Definition 3.3. By $N .^{B} \mathscr{U}$ we mean the realization of the simplicial complex $\Sigma_{\mathscr{U}}^{B}$, whose simplices are the subsets $\left\{\beta_{1}, \ldots, \beta_{k}\right\} \subseteq B$ so that $U_{\beta_{1}} \cap \ldots \cap U_{\beta_{k}} \neq \emptyset$. We define $\mathscr{E} \cdot B \mathscr{U}$ to be the realization of the simplicial complex $\mathscr{T}_{\mathscr{U}}^{B}$, whose vertex set is $B$, and so that any finite subset $\left\{\beta_{1}, \ldots, \beta_{k}\right\} \subseteq B$ is a simplex.

Of course, $\mathscr{E} .^{B} \mathscr{U}$ is weakly contractible. All these simplicial sets are viewed as subsimplicial sets of $\mathscr{E} \cdot \mathscr{U}=\mathscr{E} \cdot{ }^{A} \mathscr{U}$.

Lemma 3.4. Let $\mathscr{U}=\left\{U_{\alpha}\right\}_{\alpha \in A}$ be a covering of a space $X$, and let $B^{0} \subseteq B \subseteq A$ be subsets of $A$. Suppose further that for each $\beta \in B$, there is a $\beta^{0} \in B^{0}$ so that $U_{\beta} \subseteq U_{\beta^{0}}$. Then the evident inclusion $N .^{B^{0}} \mathscr{U} \rightarrow N .{ }^{B} \mathscr{U}$ is an equivalence.

Proof. Let $f: B \rightarrow B^{0}$ be any function so that $U_{\beta} \subseteq U_{f(\beta)}$, and $f \mid B^{0}=i d_{B^{0}}$. $f$ induces a map of nerves $N . f$, and it is clear that the composite

$$
N .^{B^{0}} \mathscr{U} \rightarrow N .^{B} \mathscr{U} \rightarrow N .^{B^{0}} \mathscr{U}
$$

is equal to the identity. On the other hand, the composite

$$
N .^{B} \mathscr{U} \rightarrow N .^{B^{0}} \mathscr{U} \rightarrow N .^{B} \mathscr{U}
$$

is simplicially homotopic to the identity in view of the fact that

$$
U_{\beta_{1}} \cap \ldots \cap U_{\beta_{k}} \neq \emptyset \Rightarrow U_{\beta_{1}} \cap \ldots \cap U_{\beta_{k}} \cap U_{f\left(\beta_{1}\right)} \cap \ldots \cap U_{f\left(\beta_{k}\right)} \neq \emptyset
$$

This gives the result.

Consider also the following situation. Let $\mathscr{U}=\left\{U_{\alpha}\right\}_{\alpha \in A}$ be a covering of a space $X$, and let $B \subseteq A$ be a subset. Let $W_{\mathscr{U}}(B) \subseteq X$ be the set $\bigcup_{\beta \in B} U_{\beta}$. Let $\Lambda^{B}(\mathscr{U})=\left\{W_{\alpha}\right\}_{\alpha \in A}$ be the covering of $X$ given by $W_{\alpha}=U_{\alpha}$ if $\alpha \notin B$, and 
$W_{\alpha}=W_{\mathscr{U}}(B)$ if $\alpha \in B$. Let $P(B)=P_{\mathscr{U}}(B) \subseteq A$ be the set of all $\alpha \in A$ so that $U_{\alpha} \cap W(B) \neq \emptyset$. We may view $\Sigma_{\Lambda^{B}(\mathscr{U})}$ as a subcomplex of $\mathscr{T}_{\mathscr{U}}$, and as such it is contained in the subcomplex $\Sigma_{\mathscr{U}} \cup_{\Sigma_{(\mathscr{U}}^{P(B)}} \mathscr{T}_{\mathscr{U}}^{P(B)}$. This is true since it is clear from the definitions that a simplex $\left\{\alpha_{1}, \ldots, \alpha_{k}\right\}$ of $\Sigma_{\Lambda^{B}(\mathscr{U})}$ either contains a vertex in $B$, in which case $\alpha_{i} \in P(B)$ for all $i$, or it does not, in which case it is in $\Sigma_{\mathscr{U}}$. Also it is clear that

$$
\Sigma_{\mathscr{U}} \cup_{\Sigma_{\mathscr{U}}^{B}} \mathscr{T}_{\mathscr{U}}^{B} \subseteq \Sigma_{\Lambda^{B}(\mathscr{U})}
$$

Lemma 3.5. Let $\mathscr{U}, A$, and $B$ be as above. Suppose that for any $\left\{\alpha_{1}, \ldots, \alpha_{k}\right\} \subseteq P_{\mathscr{U}}(B)$,

$$
U_{\alpha_{1}} \cap \ldots \cap U_{\alpha_{k}} \neq \emptyset \Rightarrow U_{\alpha_{1}} \cap \ldots \cap U_{\alpha_{k}} \cap W_{\mathscr{U}}(B) \neq \emptyset
$$

Then the inclusion

$$
\Sigma_{\Lambda^{B}(\mathscr{U})} \hookrightarrow \Sigma_{\mathscr{U}} \cup_{\Sigma_{\mathscr{U}}^{P(B)}} \mathscr{T}_{\mathscr{U}}^{P(B)}
$$

induces an equivalence on nerves.

Proof. We first note that $\Sigma_{\Lambda^{B}(\mathscr{U})}$ is the union of $\Sigma_{\mathscr{U}}$ with $\Sigma_{\Lambda^{B}(\mathscr{U})}^{P(B)}$; the overlap is $\Sigma_{\mathscr{U}}^{P(B)}$. Consequently, we have a map of pushout diagrams

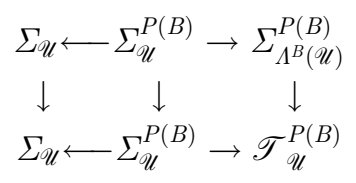

of simplicial complexes. Since the realization and nerve constructions preserve pushouts, it will suffice to show that $\Sigma_{\Lambda^{B}(\mathscr{U})}^{P(B)} \rightarrow \mathscr{T}_{\mathscr{U}}^{P(B)}$ induces a weak equivalence on nerves. Since $N . \mathscr{T}_{\mathscr{I}}^{P(B)}$ is contractible, it will suffice to show that $N . \Sigma_{\Lambda^{B}(\mathscr{U})}^{P_{\mathscr{U}}(B)}$ is contractible. Write $P_{\mathscr{U}}(B)=B \amalg Q(B)$. A typical simplex in $\Sigma_{\Lambda^{B}(\mathscr{U})}^{P_{u}(B)}$ is of the form $\left\{\beta_{1}, \ldots, \beta_{s}, q_{1}, \ldots, q_{t}\right\}$, with $\beta_{i} \in B$ and $q_{i} \in Q(B)$. Clearly $\left\{\beta_{1}, \ldots, \beta_{s}, q_{1}, \ldots, q_{t}\right\}$ is a simplex of $\Sigma_{\Lambda_{u}(B)}^{P_{u}(B)}$ if and only if $\left\{q_{1}, \ldots, q_{t}\right\}$ is a simplex in $\Sigma_{\mathscr{U}}^{Q(B)}$. Consequently $\Sigma_{\Lambda^{B}(\mathcal{U})}^{P_{\mathcal{U}}(B)}$ is the join of $\Sigma_{\Lambda^{B}(\mathcal{U})}^{Q(B)}$ with $\Sigma_{\Lambda^{B}(\mathscr{U})}^{B}$. Since $\Sigma_{\Lambda^{B}(\mathscr{U})}^{B}=\mathscr{T}_{\mathscr{U}}^{B}$, whose nerve is contractible, the result follows.

We wish to consider subspaces also. A relative covering of a subspace $Y \subseteq X$ is a parameterized family $\mathscr{U}=\left\{U_{\alpha}\right\}_{\alpha \in A}$ of open subsets $U_{\alpha}$ of $X$ so that $Y \subseteq \bigcup_{\alpha \in A} U_{\alpha} . \Sigma_{\mathscr{U}}$ and $N . \mathscr{U}$ are defined precisely as before, i.e. the simplices of $\Sigma_{\mathscr{U}}$ are finite subsets $\left\{\alpha_{1}, \ldots, \alpha_{k}\right\}$ so that $U_{\alpha_{1}} \cap \ldots \cap U_{\alpha_{k}} \neq \emptyset$. For any relative covering $\mathscr{U}$ of $Y \subseteq X$, we have the covering $\rho \mathscr{U}$ of $Y$, where $\rho \mathscr{U}=\left\{U_{\alpha} \cap Y\right\}_{\alpha \in A}$. There is an evident map $\Sigma_{\rho \mathscr{U}} \rightarrow \Sigma_{\mathscr{U}}$ given by 
$\left\{\alpha_{1}, \ldots, \alpha_{k}\right\} \rightarrow\left\{\alpha_{1}, \ldots, \alpha_{k}\right\}$, and hence a map of simplicial sets $N$. $\rho \mathscr{U} \rightarrow N$. U.

Lemma 3.6. Suppose $X$ is a compact Hausdorff space and $Y$ is a closed subspace. Let $\mathscr{U}=\left\{U_{\alpha}\right\}_{\alpha \in A}$ be a finite open covering of $Y$. Suppose further that for each $\alpha$, we are given a closed set $W_{\alpha} \subseteq U_{\alpha}$ and that $\left\{W_{\alpha}\right\}_{\alpha \in A}$ is also a covering of $Y$. Then there is a relative covering of $Y$ in $X,\left\{V_{\alpha}\right\}_{\alpha \in A}$, so that $W_{\alpha} \subseteq Y \cap V_{\alpha} \cap U_{\alpha}$, and so that for every subset $\left\{\alpha_{1}, \ldots, \alpha_{k}\right\} \subseteq A$,

$$
V_{\alpha_{1}} \cap \ldots \cap V_{\alpha_{k}}=\emptyset \Leftrightarrow W_{\alpha_{1}} \cap \ldots \cap W_{\alpha_{k}}=\emptyset
$$

Proof. This is a straightforward generalization of the fact that in a compact Hausdorff space, any two disjoint closed subspaces are contained in disjoint open sets. We leave the proof to the reader.

\section{Rigid Coverings and Čech Homology.}

In our construction of Čech homology, it will be important that the category of coverings used as parameter category for certain homotopy inverse limits is a left directed partially ordered set. We will use an analogue to the "rigid coverings" used by Friedlander [9] in his construction of the "etale topological type " associated to a scheme.

Definition 4.1. Let $X$ be a topological space. A rigid covering of $X$ is a function $\beta$ from the underlying set of $X$ to the collection of open subsets of $X$ satisfying the following three conditions.

a) $x \in \beta(x)$

b) For any open set $U \subseteq X, \overline{\beta^{-1} U} \subseteq U$

c) Only finitely many distinct open sets occur among the sets $\beta(x)$. That is, the image of $\beta$ is a finite collection of the collection of open subsets of $X$.

Let $\mathrm{RC}(X)$ denote the set of all rigid coverings of $X$. If $\beta_{1}, \beta_{2} \in \mathrm{RC}(X)$, we say $\beta_{1}$ refines $\beta_{2}$ and write $\beta_{1} \geq \beta_{2}$ if and only if $\beta_{1}(x) \subseteq \beta_{2}(x)$ for all $X$, $\mathrm{RC}(X)$ now becomes a partially ordered set, and hence can be viewed as a category. We also define a relative version, where $Y \subseteq X$ is a subspace. A relative rigid covering of $Y$ in $X$ is a function $\beta$ from the underlying set of $Y$ into the open subsets of $X$, which satisfies properties a)-c) above. We similarly obtain a category $\mathrm{RC}(X, Y)$.

Thus, a rigid covering is an open covering of $X$, with parameter set $X$, so that only finitely many distinct subsets occur. It turns out that $X \rightarrow \mathrm{RC}(X)$ defines a contravariant functor from the category of pointed topological spaces to the category of pointed small categories. 
Proposition 4.2. For any map of topological spaces $f: X \rightarrow Y$, and $\beta \in R C(Y)$, we define a function $f^{!} \beta$ from the underlying set of $X$ into the collection of open subsets of $X$ by the formula $f^{!} \beta(x)=f^{-1} \beta(f(x))$. Then $f^{!} \beta$ is a rigid covering of $X$, and the formulae $X \rightarrow R C(X)$ and $f \rightarrow f^{!}$make $R C(-)$ into a contravariant functor.

Proof. All conditions defining $\mathrm{RC}(X)$ are clear except $\mathrm{b})$. To check $\mathrm{b})$, let $U$ be an open subset occurring in the image of $\beta$, i.e. $U=\beta(y)$. Then $f^{!^{-1}}\left(f^{-1} U\right)$ is equal to the union of the inverse images under $f$ of all the sets $\beta^{-1} V$, as $V$ ranges over all the sets in the image of $\beta$ for which $f^{-1} V=f^{-1} U$. Note that this is clearly a finite union, and the closure of each set $f^{-1} V$ is contained in $f^{-1} U$. Since for a finite union,

$$
\overline{V_{1}} \cup \ldots \cup \overline{V_{k}}=\overline{V_{1} \cup \ldots \cup V_{k}}
$$

condition b) follows.

We will also need a kind of product of rigid coverings.

Proposition 3. Let $\beta_{1}$ and $\beta_{2}$ denote rigid coverings of a space $X$. We define a new function $\beta_{1} \times_{X} \beta_{2}$ from the set $X$ into the collection of open subsets of $X$ by the formula $\beta_{1} \times_{X} \beta_{2}(x)=\beta_{1}(x) \cap \beta_{2}(x)$. Then $\beta_{1} \times_{X} \beta_{2}$ is a rigid covering of $X$. Furthermore, it refines both $\beta_{1}$ and $\beta_{2}$.

Proof. As in the preceding proposition, all is clear except the fact that $\beta_{1} \times_{X} \beta_{2}$ satisfies condition $b$ ) in the definition of rigid coverings. To check this condition, we construct first the function $\beta_{1} \times \beta_{2}$ from $X \times X$ into the collection of open subsets of $X \times X$ by the formula

$$
\left(\beta_{1} \times \beta_{2}\right)\left(x_{1}, x_{2}\right)=\beta_{1}\left(x_{1}\right) \times \beta_{2}\left(x_{2}\right)
$$

$\beta_{1} \times \beta_{2}$ is evidently a rigid covering of $X \times X$. Now, if we let $\Delta: X \rightarrow X \times X$ denote the diagonal map, then

$$
\beta_{1} \times{ }_{X} \beta_{2}=\Delta^{!} \beta_{1} \times \beta_{2}
$$

and the result follows directly from 2 .

It thus follows that $\mathrm{RC}(X)$ is a left directed partially ordered set. Given an arbitrary covering $\mathscr{U}=\left\{U_{\alpha}\right\}_{\alpha \in A}$, the reader may wonder if there is a rigid covering $\beta$ of $X$, so that for all $x \in X, \beta(x)=U_{\alpha(x)}$ for some $\alpha(x)$. Indeed, this question is important for us for technical reasons. The following lemma will be useful.

Lemma 4. Given any covering $\mathscr{U}=\left\{U_{\alpha}\right\}_{\alpha \in A}$ of a compact Hausdorff space, there is a rigid covering $\beta$ of $X$ so that for each $x \in X$, there is an $\alpha(x) \in A$ so that $\beta(x)=U_{\alpha(x)}$. 
Proof. Since $X$ is compact, we may assume that $A$ is finite. Since $X$ is compact Hausdorff, there is a covering $\left\{V_{\alpha}\right\}_{\alpha \in A}$, with $\overline{V_{\alpha}} \subseteq U_{\alpha}$ for all $\alpha$. Let $\phi: X \rightarrow A$ be such that $x \in V_{\phi(x)}$ for all $x$. Then define $\beta$ by $\beta(x)=U_{\phi(x)}$. This gives the required rigid covering.

Lemma 4.5. For every $\beta \in R C(X)$ and closed subset $Y$, there is an open set $U$, with $Y \subseteq U$, and a refinement $\beta^{*} \in \operatorname{RC}(X), \beta^{*} \geq \beta$, so that for every $x \in U, \beta^{*}(x) \subseteq \beta(y)$ for some $y \in Y$.

Proof. Consider $\beta \mid Y \in \mathrm{RC}(X, Y)$. We first construct an open set $V$ and $\tilde{\beta} \in \operatorname{RC}(X)$ so that $\tilde{\beta}|Y=\beta| Y$, and so that for all $x \in V, \tilde{\beta}(x) \subseteq \tilde{\beta}(y)$ for some $y$. Let $U=\bigcup_{y \in Y} \beta(y)$. This is an open set containing $Y$. Since $X$ is compact Hausdorff, there is an open set $V$, with $Y \subseteq V \subseteq \bar{V} \subseteq U$. Consider the open covering consisting of all the sets $\beta(y)$ together with $X-\bar{V}$. Again since $X$ is compact Hausdorff, we may select an open covering $\left\{Z_{y}\right\}_{y \in Y} \cup\{W\}$ so that $\bar{Z}_{y} \subseteq \beta(y)$ and $\bar{W} \subseteq X-\bar{V}$. For every $x \in X-Y$, choose $\tilde{\beta}(x)$ to be either (i.) $\beta(y)$, where $y$ is such that $x \in Z_{y}$, or (ii.) $X-\bar{V}$ if $x \in W$. (Note that both possibilities can occur simultaneously, so the construction of $\beta^{*}$ involves choices.) If $x \in Y$, set $\tilde{\beta}(x)=\beta(x)$. With this choice of $V$ and $\tilde{\beta}$, we clearly have that if $x \in V, \tilde{\beta}(x) \subseteq \beta(y)$ for some $y \in Y$. Now set $\beta^{*}=\tilde{\beta} \times_{X} \beta$. With the same choice of $V$, this clearly has the required properties.

Lemma 4.6. Let $X$ be a compact Hausdorff space, and $Y \subseteq X$ a closed subspace. Let $\beta \in \mathrm{RC}(X, Y)$. Then there is a rigid covering $\hat{\beta} \in \mathrm{RC}(X)$ so that $\hat{\beta} \mid Y=\beta$.

Proof. Let $Z$ denote the open set $\bigcup_{y \in Y} \beta(y) \subseteq X$. Of course, $Y \subseteq Z$. Since $X$ is compact Hausdorff and $Y$ is closed, we may choose an open set $V$, so that $V \supseteq X-Z$, and so that $\bar{V} \cap Y=\emptyset$. Let $\left\{U_{\alpha}\right\}_{\alpha \in A}$ be an indexing of the sets which occur in the image of $\beta$, together with $V$, so $A$ is finite. Since $X$ is compact Hausdorff and $Y$ is closed, there is a family of open sets $\left\{W_{\alpha}\right\}_{\alpha \in A}$ with $\overline{W_{\alpha}} \subseteq U_{\alpha}$ and $W_{\alpha} \supseteq \beta^{-1}\left(U_{\alpha}\right)$ for all $\alpha \in A$, and so that $\left\{W_{\alpha}\right\}_{\alpha \in A}$ is a covering of $X$. For each $y \in Y$, set $\hat{\beta}(y)=\beta(y)$. For each $x \in X-Y$, find an $\alpha \in A$ so that $x \in U_{\alpha}$, and set $\hat{\beta}(x)=U_{\alpha}$. This construction gives the required rigid covering.

Lemma 4.7. Let $X$ be a compact Hausdorff space, and let $Y \subseteq X$ be a closed subspace. Let $\beta$ be any rigid covering. Then there is a rigid covering $\beta^{*} \geq \beta$, so that

$$
\beta^{*}\left(x_{1}\right) \cap \ldots \cap \beta^{*}\left(x_{k}\right)=\emptyset \Rightarrow \overline{\beta^{*}\left(x_{1}\right)} \cap \ldots \cap \overline{\beta^{*}\left(x_{k}\right)}=\emptyset
$$


for all $\left\{x_{1}, \ldots, x_{k}\right\}$. Further, $\beta^{*}$ can be chosen so that

$$
\beta^{*}\left(x_{1}\right) \cap \ldots \cap \beta^{*}\left(x_{k}\right) \cap Y=\emptyset \Rightarrow \overline{\beta^{*}\left(x_{1}\right)} \cap \ldots \cap \overline{\beta^{*}\left(x_{k}\right)} \cap Y=\emptyset
$$

Proof. Let $\left\{U_{\alpha}\right\}_{\alpha \in A}$ be a finite listing of all the subsets of the form $\beta(x)$ for some $x \in X$. Let $V_{\alpha} \subseteq U_{\alpha}$ be $\overline{\beta^{-1}\left(U_{\alpha}\right)} \subseteq U_{\alpha}$. Since $X$ is compact Hausdorff, we can choose open sets $W_{\alpha}$, so that $V_{\alpha} \subseteq W_{\alpha} \subseteq \overline{W_{\alpha}} \subseteq U_{\alpha}$, and define a new rigid covering $\beta^{(1)}$ by $\beta^{(1)}(x)=W_{\alpha}$ if and only if $\beta(x)=U_{\alpha}$. Note that

$$
\beta\left(x_{1}\right) \cap \ldots \cap \beta\left(x_{k}\right) \cap Y=\emptyset \Rightarrow \overline{\beta^{(1)}\left(x_{1}\right)} \cap \ldots \cap \overline{\beta^{(1)}\left(x_{k}\right)} \cap Y=\emptyset
$$

Note also that $\mathscr{W}=\left\{W_{\alpha}\right\}_{\alpha \in A}$ is now a finite covering, and the identity map on $A$ produces an inclusion of finite simplicial complexes $\Sigma_{\mathscr{W}} \hookrightarrow \Sigma_{\mathscr{U}}$. Repeating this process gives a descending chain of finite simplicial complexes on the same vertex set $A$, which must eventually stabilize. This means that we have open sets $W_{\alpha}^{\prime}$ and $W_{\alpha}$, with

$$
V_{\alpha} \subseteq W_{\alpha}^{\prime} \subseteq \overline{W_{\alpha}^{\prime}} \subseteq W_{\alpha} \subseteq \overline{W_{\alpha}} \subseteq U_{\alpha}
$$

so that

$$
W_{\alpha_{1}}^{\prime} \cap \ldots \cap W_{\alpha_{k}}^{\prime}=\emptyset \Rightarrow W_{\alpha_{1}} \cap \ldots \cap W_{\alpha_{k}}=\emptyset
$$

which implies that $\overline{W_{\alpha_{1}}^{\prime}} \cap \ldots \cap \overline{W_{\alpha_{k}}^{\prime}}=\emptyset$. Define $\beta^{*}$ by $\beta^{*}(x)=W_{\alpha}^{\prime}$ if and only if $\beta(x)=U_{\alpha}$.

Definition 4.8. Let $\left(X, x_{0}\right)$ be a topological space, and let $\Phi_{X}$ be the functor from $\mathrm{RC}(X)$ to $s \mathscr{S}$ given by $\beta \rightarrow N$. $\beta$. For any homology theory $T: s \mathscr{S} \rightarrow \mathscr{K}$ from based simplicial sets to based Kan complexes, we define the Čech homology of $X$ with "coefficients in $T$ ", $\breve{h}\left(X, x_{0} ; T\right)$, to be

$$
\underset{\operatorname{RC}(X)}{\operatorname{holim}} T \circ \Phi_{X}
$$

This defines $\check{h}$ on objects. If $f: X \rightarrow Y$ is a map of topological spaces, we define $\breve{h}(f ; T)$ to be the composite

$$
\underset{\mathrm{RC}(X)}{\operatorname{holim}} T \circ \Phi_{X} \rightarrow \underset{\mathrm{RC}(Y)}{\operatorname{holim}} T \circ \Phi_{X} \circ R C(f) \rightarrow \underset{\mathrm{RC}(Y)}{\operatorname{holim}} T \circ \Phi_{Y}
$$

where the left arrow is pullback of homotopy inverse limits along the functor $\mathrm{RC}(f)$ and where the right hand arrow is induced by the evident natural transformation $\Phi_{X} \circ R C(f) \rightarrow \Phi_{Y}$.

Notice that the basepoint $x_{0} \in X$ determines a basepoint in $\breve{h}\left(X, x_{0} ; T\right)$, so $\check{h}(-; T)$ can be viewed as a functor from the category of based spaces to $\mathscr{K}$. We will occasionally suppress the basepoint when no confusion will result. 


\section{Excision.}

Throughout this section, let $X$ denote a compact Hausdorff space and let $Y \subseteq X$ denote a closed subspace. Let $T$ denote a functor from the category of based simplicial sets to the category of based Kan complexes which is a homology theory in the sense of Definition 2.1. Let $X$ be any compact Hausdorff space, and let $Y \subseteq X$ be a closed subspace. In this section, we will prove "strong excision" for the functor $\breve{h}(-; T)$, i.e. that the sequence of maps

$$
\breve{h}\left(Y, y_{0} ; T\right) \rightarrow \check{h}\left(X, x_{0} ; T\right) \rightarrow \breve{h}\left(X / Y,\left[y_{0}\right] ; T\right)
$$

is a fibration up to homotopy in the sense that the evident map from $\breve{h}\left(Y, y_{0} ; T\right)$ to the homotopy fiber of the $\operatorname{map} \breve{h}\left(X, x_{0} ; T\right) \rightarrow \breve{h}\left(X / Y,\left[y_{0}\right] ; T\right)$ is an equivalence of Kan complexes.

We first observe that for any based pair of spaces $(X, Y)$, we have a commutative diagram

$$
\begin{array}{ccc}
\check{h}\left(Y, y_{0} ; T\right) & \rightarrow & \breve{h}(*, * ; T) \\
\downarrow & \downarrow & \downarrow \\
\check{h}\left(X, x_{0} ; T\right) & \rightarrow & h(X / Y, * ; T)
\end{array}
$$

THeOREm 5.1. Let $X$ be a compact Hausdorff space, and $Y \subseteq X$ a closed subspace. Then the above diagram is homotopy Cartesian.

Proof. The strategy will be to find a weakly equivalent diagram which is induced by a diagram of functors over $\mathrm{RC}(X)$, and to apply 2.5 suitably. Let $i: Y \hookrightarrow X$ and $j: * \hookrightarrow X / Y$ denote the inclusions and let $p: X \rightarrow X / Y$ and $q: Y \rightarrow$ denote the projections onto the quotient space. The above diagram may be written as follows.

$$
\begin{array}{lll}
\underset{\operatorname{RC}(Y)}{\operatorname{holim}} & T \circ \Phi_{Y} \rightarrow \operatorname{holim}_{\mathrm{RC}(*)} & T \circ \Phi_{*} \\
& & \\
& \downarrow & \downarrow \\
\operatorname{holim} & T \circ \Phi_{X} \rightarrow \operatorname{holim} & T \circ \Phi_{X / Y} \\
\operatorname{RC}(X) & &
\end{array}
$$

It follows from Lemma 4.6 that the conditions of Proposition 2.4 are satisfied, so this diagram is weakly equivalent to the new diagram 
(A)

$$
\begin{aligned}
& \underset{\mathrm{RC}(X)}{\text { holim }} \quad T \circ \Phi_{Y} \circ \mathrm{RC}(i) \rightarrow \operatorname{holim}_{\mathrm{RC}(X Y)} \quad T \circ \Phi_{*} \circ R C(j) \\
& \underset{\operatorname{RC}(X)}{\operatorname{holim}} \stackrel{\downarrow}{T \circ \Phi_{X} \rightarrow \operatorname{holim}} \underset{\operatorname{RC}(X / Y)}{ } \stackrel{\downarrow}{T \circ} \Phi_{X / Y}
\end{aligned}
$$

where the vertical arrows are induced by natural transformations $\Phi_{Y} \circ R C(i) \rightarrow \Phi_{X}$ and $\Phi_{*} \circ R C(j) \rightarrow \Phi_{X / Y}$. The horizontal arrows are pullback maps along $\mathrm{RC}(p)$ composed with maps induced by the natural transformation from $\Phi_{Y} \circ R C(i) \circ R C(p)=\Phi_{Y} \circ R C(q) \circ R C(j) \rightarrow \Phi_{*} \circ R C(j)$ and $\Phi_{X} \circ R C(p) \rightarrow \Phi_{X / Y}$. Exhibiting only the natural transformations we get the diagram of functors and natural transformations

$$
\begin{gathered}
\Phi_{Y} \circ R C(p \circ i) \rightarrow \Phi_{*} \circ R C(j) \\
\downarrow \\
\Phi_{X} \circ R C(p) \rightarrow \Phi_{X / Y}
\end{gathered}
$$

Denoting the functor $\mathrm{RC}(Z) \rightarrow s \mathscr{S}$ sending $\beta$ to $R . \mathscr{T}_{\beta}$ by $C_{Z}$ for any space $Z$, and the constant functor with value the one point simplicial set by $\mathscr{E}$, this diagram of natural transformations factors as described in the following diagram.

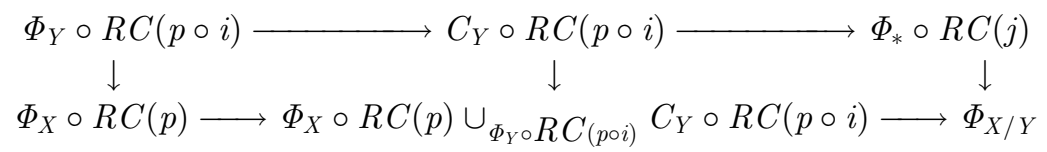

and consequently we have a map of diagrams from the diagram $(B)$ below to diagram $(A)$.

$$
\begin{aligned}
& \underset{\mathrm{RC}(X)}{\operatorname{holim}} \quad \Phi_{Y} \circ \mathrm{RC}(i) \longrightarrow \operatorname{holim}_{\mathrm{RC}(X)} C_{Y} \circ \mathrm{RC}(i) \\
& \underset{\operatorname{RC}(X)}{\operatorname{holim}} \Phi_{X} ! \longrightarrow \operatorname{holim}_{\operatorname{RC}(X / Y)} \Phi_{X} \cup_{\Phi Y \circ R C(i)} C_{Y} \circ R C(i)
\end{aligned}
$$

The maps on the left hand part of the diagram are identity maps, and the map in the upper right hand corner is an equivalence since both entries are contractible. Further, the last diagram is homotopy Cartesian in view of Proposition 2.5. Consequently, it suffices to show that the map

$$
\underset{R C(X)}{\operatorname{holim}} T \circ\left(\Phi_{X} \cup_{\Phi_{Y} \circ R C(i)} C_{Y} \circ R C(i)\right) \rightarrow \operatorname{holim}_{R C(X / Y)} T \circ \Phi_{X / Y}
$$

is an equivalence. The proof of this result is in two stages, since this map is actually a composite of two maps, one 


$$
\begin{aligned}
& \operatorname{holim}_{\beta \in R C(X)} T\left(\Phi_{X}(\beta) \cup_{\Phi_{Y} \circ R C(i)(\beta)} C_{Y} \circ R C(i)(\beta)\right) \rightarrow \\
& \operatorname{holim}_{\beta \in R C(X / Y)} T\left(\Phi_{X} \circ R C(p)(\beta) \cup_{\Phi_{Y} \circ R C(p \circ i)(\beta)} C_{Y} \circ R C(p \circ i)(\beta)\right)
\end{aligned}
$$

and the other

$$
\operatorname{holim}_{\beta \in R C(X / Y)} T\left(\Phi_{X} \circ R C(p)(\beta) \cup_{\Phi_{Y} \circ R C(p \circ i)(\beta)} C_{Y} \circ R C(p \circ i)(\beta)\right) \rightarrow \operatorname{holim}_{R C(X / Y)} \Phi_{X / Y} .
$$

The first is restriction along the inclusion $\operatorname{RC}(p)$, and the second is induced by a natural transformation.

We analyze the restriction map first. For any $\beta \in \mathrm{RC}(X)$, let $\beta^{0}$ be the rigid covering of $Y$ given by $\beta^{0}(y)=\beta(y) \cap Y$. Thus, $\Phi_{Y} \circ R C(i)(\beta)=\beta^{0}$. We have a natural inclusion of simplicial complexes $\Sigma_{\beta^{0}} \hookrightarrow \Sigma_{\beta}^{Y}$. We will show first that for every $\beta \in \mathrm{RC}(X)$, there is a $\bar{\beta} \geq \beta$ so that the natural inclusion $N . \bar{\beta}^{0} \rightarrow N . \bar{\beta}$ is an equivalence. To construct $\bar{\beta}$, let $\left\{U_{\alpha}\right\}_{\alpha \in A}$ be a listing, with finite index set, of the distinct subsets occurring in the image of $\beta^{0} \mid Y$, and let $W_{\alpha} \subseteq U_{\alpha}$ be the closed sets $\overline{\left(\beta^{0}\right)^{-1}\left(U_{\alpha}\right)}$. According to 3.6, there exist open sets $V_{\alpha}$ in $X$ so that $W_{\alpha} \subseteq V_{\alpha} \cap Y \subseteq U_{\alpha}$, and so that

$$
V_{\alpha_{1}} \cap \ldots \cap V_{\alpha_{k}} \neq \emptyset \Rightarrow W_{\alpha_{1}} \cap \ldots \cap W_{\alpha_{k}} \neq \emptyset
$$

Let $\tilde{\beta} \in \operatorname{RC}(X, Y)$ be defined by $\tilde{\beta}(y)=V_{\alpha}$ if and only if $\beta^{0}(y)=U_{\alpha}$. Then by 4.6 , it is possible to find a rigid covering $\hat{\beta}$ of $X$, with $\hat{\beta} \mid Y=\tilde{\beta}$. $\bar{\beta}$ will now be taken to be $\beta \times_{X} \hat{\beta} . \beta \times_{X} \hat{\beta} \mid Y=\tilde{\beta}$, and from the construction of $\tilde{\beta}$, it is clear that the map $\Sigma_{\bar{\beta}^{0}} \rightarrow \Sigma_{\bar{\beta}}^{Y}$ is an isomorphism of simplicial complexes. This is the required result. From the definition of homotopy pushouts, it follows that the natural map

$$
\operatorname{holim}_{\beta \in R C(X)} T\left(R . \Sigma_{\beta} \cup_{R . \Sigma_{\beta} 0} R . \mathscr{T}_{\beta} Y\right) \rightarrow \operatorname{holim}_{\beta \in R C(X)} T\left(R . \Sigma_{\beta} \cup_{R . \Sigma_{\beta}^{Y}} R . \mathscr{T}_{\beta}^{Y}\right)
$$

is an equivalence. Further, it is clear that if $\beta \in \mathrm{RC}(X / Y), \Sigma_{\beta^{0}}=\Sigma_{\beta}^{Y}$, and it will therefore suffice to show that the restriction map

$$
\operatorname{holim}_{\beta \in R C(X)} T\left(R . \Sigma_{\beta} \cup_{\left.R . \Sigma_{\beta}^{Y}\right)} R . \mathscr{T}_{\beta}^{Y}\right) \underset{\beta \in R C(X / Y)}{\rightarrow} \operatorname{holim} T\left(R . \Sigma_{\beta} \cup_{R . \Sigma_{\beta}^{Y}} R . \mathscr{T}_{\beta}^{Y}\right)
$$

is an equivalence. We will show this via a series of equivalences. We have a commutative diagram

$$
\begin{aligned}
& \begin{array}{lcc}
\operatorname{holim} & T\left(R . \Sigma_{\beta} \cup_{R . \Sigma_{\beta}^{Y}} R . \mathscr{T}_{\beta}^{Y}\right) \longrightarrow \operatorname{holim}_{\beta \in R C(X)} & T\left(R . \Sigma_{\beta} \cup_{\Sigma_{\beta}^{W(Y)}} R . \mathscr{T}_{\beta}^{W(Y)}\right) \\
\downarrow & \downarrow & \downarrow
\end{array} \\
& \text { holim } T\left(R . \Sigma_{\beta} \cup_{R . \Sigma_{\beta}^{Y}} R . \mathscr{T}_{\beta}^{Y}\right) \rightarrow \operatorname{holim} T\left(R . \Sigma_{\beta} \cup_{\Sigma_{\beta}^{W(Y)}} R . \mathscr{T}_{\beta}^{W(Y)}\right) \\
& \beta \in R C(X / Y) \quad \beta \in R C(X / Y)
\end{aligned}
$$

where the horizontal arrows are induced by natural inclusions of simplicial 
complexes, and where the vertical arrows are restriction maps. We will show that the horizontal arrows are equivalences. It will then follow that if the right hand vertical arrow is an equivalence, then so is the left hand arrow, and we will then proceed to show that the right hand vertical arrow is an equivalence.

To show that the upper horizontal arrow is an equivalence, it will suffice, in view of the fact that $T$ is a homology theory and that $R . \mathscr{T}_{\beta}^{Y}$ and $R . \mathscr{T}_{\beta}^{W(Y)}$ are contractible, to show that

$$
\underset{\beta \in R C(X)}{\operatorname{holim}} T\left(R . \Sigma_{\beta}^{Y}\right) \rightarrow \underset{\beta \in R C(X)}{\operatorname{holim}} T\left(R . \Sigma_{\beta}^{W(Y)}\right)
$$

is an equivalence. In view of 4.5 , for any $\beta \in \mathrm{RC}(X)$, there is a $\beta^{*} \geq \beta$, so that for any $x \in W(Y)$, there is a $y \in Y$ so that $\beta^{*}(x) \subseteq \beta^{*}(y)$. From 3.4, it follows that the inclusion $\Sigma_{\beta}^{Y} \rightarrow \Sigma_{\beta}^{W(Y)}$ induces a weak equivalence on nerves, and the result now follows from 2.6. To see that the lower horizontal arrow is an equivalence, it similarly suffices to show that $\operatorname{holim}_{\beta \in R C(X / Y)} T\left(R . \Sigma_{\beta}^{Y}\right) \rightarrow \operatorname{holim}_{\beta \in R C(X / Y)} T\left(R . \Sigma_{\beta}^{W(Y)}\right)$ is an equivalence. This follows as above with the additional observation that we may take $\beta^{*}$ to lie in $\mathrm{RC}(X / Y)$.

In order to prove that the restriction map

$$
\underset{\beta \in R C(X)}{\operatorname{holim}} T\left(R . \Sigma_{\beta} \cup_{\Sigma_{\beta}^{W(Y)}} \mathscr{T}_{\beta}^{W(Y)}\right) \underset{\beta \in R C(X / Y)}{\rightarrow \operatorname{holim}} T\left(R . \Sigma_{\beta} \cup_{\Sigma_{\beta}^{W(Y)}} \mathscr{T}_{\beta}^{W(Y)}\right)
$$

is an equivalence, consider the following commutative diagram.

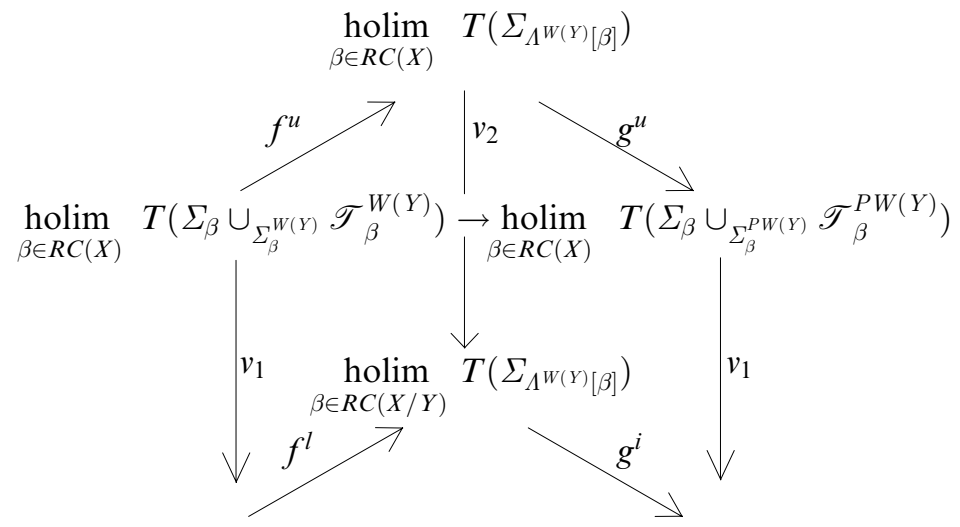

$\operatorname{holim}_{\beta \in R C(X / Y)} T\left(\Sigma_{\beta} \cup_{\Sigma_{\beta}^{W(Y)}} \mathscr{T}_{\beta}^{W(Y)}\right) \stackrel{h^{l}}{\rightarrow} \operatorname{holim}_{\beta \in R C(X / Y)} T\left(\Sigma_{\beta} \cup_{\Sigma_{\beta}^{P W(Y)}} \mathscr{T}_{\beta}^{P W(Y)}\right)$

The triangles at either end of the "prism" are induced by natural inclusions of simplicial complexes, as described in section 5. The vertical arrows are all 
restriction maps. We wish to show that $v_{1}$ is an equivalence; for this it will suffice to show that $f^{u}, f^{l}$, and $v_{2}$ are equivalences. We first deal with $f^{u}$. From the diagram, it will clearly suffice to show that $h^{u}$ and $g^{u}$ are equivalences. To show that $h^{u}$ is an equivalence, we observe that in view of the fact that $T$ is a homology theory and the contractibility of $\mathscr{T}_{\beta}^{W(Y)}$ and $\mathscr{T}_{\beta}^{P W(Y)}$ it will suffice to show that the inclusion

$$
\operatorname{holim}_{\beta \in R C(X)} T\left(R . \Sigma_{\beta}^{W(Y)}\right) \rightarrow \underset{\beta \in R C(X)}{\operatorname{holim}} T\left(R . \Sigma_{\beta}^{P W(Y)}\right)
$$

is an equivalence. Since $T$ is a homology theory, it will suffice to show that

$$
\operatorname{holim}_{\beta \in R C(X)} T\left(R . \Sigma_{\beta}^{P W(Y)} / R . \Sigma_{\beta}^{W(Y)}\right)
$$

is contractible. For this, it will suffice by 2.6 to show that for every $\beta \in \mathrm{RC}(X)$, there is a $\beta^{*} \in \mathrm{RC}(X)$, with $\beta^{*} \geq \beta$, so that the map

$$
\text { R. } \Sigma_{\beta^{*}}^{P(Y)} / R . \Sigma_{\beta^{*}}^{W(Y)} \rightarrow R . \Sigma_{\beta}^{P W(Y)} / R . \Sigma_{\beta}^{W(Y)}
$$

is simplicially homotopic to a constant map. We first choose $\beta^{\prime} \geq \beta$, so that for every $x \in X$ with $\beta^{\prime}(x) \cap Y \neq \emptyset$, there is a $y \in Y$ with $\beta^{\prime}(x) \subseteq \beta(y)$. Now, let $U$ be an open set, with $Y \subseteq U \subseteq \bigcup_{\left\{x \mid \beta^{\prime}(x) \cap Y \neq \emptyset\right\}} \beta^{\prime}(x)$. Also, choose $V$ open, with $Y \subseteq V \subseteq \bar{V} \subseteq U$. These choices are possible since $X$ is compact Hausdorff. Consider the open covering $\{U, W-\bar{V}, X-\bar{U}\}$. Let $\gamma$ be a rigid covering of $X$ so that for each $x \in X, \gamma(x)$ is one of these sets, the existence of which is guaranteed by 4.4 , and consider $\beta^{*}=\beta^{\prime} \times_{X} \gamma$. We have $\beta^{*} \geq \beta^{\prime} \geq \beta$, and furthermore it is clear from the definitions that if we let $W^{*}=\left\{x \mid \beta^{*}(x) \cap Y \neq \emptyset\right\}$ and $\hat{W}=\left\{x \mid \beta^{*}(x) \cap W^{*} \neq \emptyset\right\}$, then $\hat{W} \subseteq W$. From this it follows that we may define a function $\phi$ from $P\left(W\left(Y, \beta^{*}\right)\right)$ to $Y$ so that $\beta^{*}(x) \subseteq \beta(\phi(y))$. We may also insist that $\phi(y)=y$ for all $y \in W\left(Y, \beta^{*}\right)$, since $\beta^{*}$ refines $\beta$. Since maps of coverings induce maps of the associated simplicial complexes, we obtain a map of simplicial complexes $\Sigma_{\beta^{*}}^{P W(Y)} \rightarrow \Sigma_{\beta}^{W(Y)}$, so that the composite

$$
\Sigma_{\beta^{*}}^{P W(Y)} \rightarrow \Sigma_{\beta}^{W(Y)} \rightarrow \Sigma_{\beta}^{P W(Y)}
$$

induces a map on realizations which is simplicially homotopic, rel $R . \Sigma_{\beta^{*}}^{W\left(Y, \beta^{*}\right)}$ to the map $R . \Sigma_{\beta^{*}}^{P W(Y)} \rightarrow R . \Sigma_{\beta}^{P W(Y)}$ induced by the identity map on $X$. This clearly gives the result, so the map $h^{u}$ is an equivalence. That

$$
h_{\beta \in R C(X / Y)}^{l}: \operatorname{holim} T\left(R . \Sigma_{\beta}^{W(Y)}\right) \rightarrow \underset{\beta \in R C(X / Y)}{\operatorname{holim} T}\left(R . \Sigma_{\beta}^{P W(Y)}\right)
$$

is an equivalence follows from the same argument, again by observing that $\beta^{*}$ may be taken to lie in $\mathrm{RC}(X / Y)$. 
We must now deal with $g^{u}$. According to 2.6 and 3.5, it will suffice to construct, for every $\beta \in \mathrm{RC}(X)$, a $\beta^{\prime} \geq \beta$, so that for any $\left\{x_{1}, \ldots, x_{k}\right\} \subseteq X$, with $\beta^{\prime}\left(x_{i}\right) \cap W\left(Y, \beta^{\prime}\right) \neq \emptyset$ for all $i$,

$$
\beta^{\prime}\left(x_{1}\right) \cap \ldots \cap \beta^{\prime}\left(x_{k}\right) \neq \emptyset \Rightarrow \beta^{\prime}\left(x_{1}\right) \cap \ldots \cap \beta^{\prime}\left(x_{k}\right) \cap W\left(Y, \beta^{\prime}\right) \neq \emptyset
$$

To construct $\beta^{\prime}$, first construct $\hat{\beta} \geq \beta$ so that

$$
\overline{\hat{\beta}\left(x_{1}\right) \cap \ldots \cap \hat{\beta}\left(x_{k}\right)} \neq \emptyset \Rightarrow \hat{\beta}\left(x_{1}\right) \cap \ldots \hat{\beta}\left(x_{k}\right) \neq \emptyset
$$

For each $\left\{x_{1}, \ldots, x_{k}\right\}$ such that $\hat{\beta}\left(x_{i}\right) \cap W(\hat{\beta}, Y) \neq \emptyset$ for all $i$, and such that $\hat{\beta}\left(x_{1}\right) \cap \ldots \hat{\beta}\left(x_{k}\right) \cap W(\hat{\beta}, Y) \neq \emptyset$, we have the closed set $\overline{\hat{\beta}}\left(x_{1}\right) \cap \ldots \cap \hat{\beta}\left(x_{k}\right)$. Since

$$
\overline{\hat{\beta}\left(x_{1}\right) \cap \ldots \cap \hat{\beta}\left(x_{k}\right)} \neq \emptyset \Rightarrow \hat{\beta}\left(x_{1}\right) \cap \ldots \cap \hat{\beta}\left(x_{k}\right)=\emptyset
$$

we see that $\overline{\hat{\beta}\left(x_{1}\right) \cap \ldots \cap \hat{\beta}\left(x_{k}\right)}$ is disjoint from $\overline{W(\hat{\beta}, Y)}$. Let $S$ be the family of all subsets $\left\{x_{1}, \ldots, x_{k}\right\}$, such that $\hat{\beta}\left(x_{i}\right) \cap W(\hat{\beta}, Y) \neq \emptyset$ for all $i$, and so that $\hat{\beta}\left(x_{1}\right) \cap \ldots \cap \hat{\beta}\left(x_{k}\right)=\emptyset$. Then

$$
Z=\bigcup_{\left\{x_{1}, \ldots, x_{k}\right\} \in S} \overline{\hat{\beta}}\left(x_{1}\right) \cap \ldots \cap \overline{\hat{\beta}}\left(x_{k}\right)
$$

is a closed set disjoint from $\overline{W(\hat{\beta}, Y)}$. Let $V=X-Z$, so $V$ is an open set containing $W(\hat{\beta}, Y)$. Choose an open set $Z_{1}$ so that

$$
\overline{W(\hat{\beta}, Y)} \subseteq Z_{1} \subseteq \overline{Z_{1}} \subseteq V
$$

and let $U=X-\overline{Z_{1}}$. Then $\{U, V\}$ is an open covering of $X$, and we let $\gamma$ be a rigid covering so that $\gamma(x)=U$ or $V$ for all $X$. Let $\beta^{\prime}=\hat{\beta} \times_{X} \gamma$. $\beta^{\prime}$ now clearly has the required properties, so $g^{u}$ is an equivalence. As usual, $g^{l}$ follows by the identical argument, with the observation that $\hat{\beta}$ and $\gamma$ may be taken to be in $\operatorname{RC}(X / Y)$. The conclusion is that $f^{u}$ and $f^{l}$ are equivalences. Thus, for our purposes, it will suffice to show that $v_{2}$ is an equivalence. But this follows directly from 2.8 , since it is easily checked that $\Lambda^{W(Y)} \beta$ is an object of $\mathrm{RC}(X / Y)$ for all $\beta$. The point is that for any $x$ so that $\left(\Lambda^{W(Y)} \beta\right)(x) \cap Y \neq \emptyset, Y \subseteq\left(\Lambda^{W(Y)} \beta\right)(x)$, so if we let $\beta^{r}$ be the rigid covering of $X / Y$ given by $\beta^{r}([x])=p\left(\left(\Lambda^{W(Y)} \beta\right)(x)\right)$, then $\Lambda^{W(Y)} \beta=R C(p)\left(\beta^{r}\right)$.

Finally, then, we must show that the natural transformation

$$
\Phi_{X} \circ R C(p) \cup_{\Phi_{Y} \circ R C(p \circ i)} C_{Y} \circ R C(p \circ i) \rightarrow \Phi_{X / Y}
$$

is a weak equivalence of functors. To see this, we note that the inclusion

$$
\Phi_{X} \circ R C(p) \rightarrow \Phi_{X} \circ R C(p) \cup_{\Phi_{Y} \circ R C(p \circ i)} C_{Y} \circ R C(p \circ i)
$$

is a weak equivalence of functors, since $\Phi_{Y} \circ R C(p \circ i)(\beta)$ is evidently con- 
tractible for each $\beta \in \mathrm{RC}(X / Y)$. We claim $\Phi_{X} \circ R C(p) \rightarrow \Phi_{X / Y}$ is a weak equivalence of functors. But this is clear from 3.2, since $\Phi_{X} \circ R C(p)(\beta)$ is the covering obtained from $\Phi_{X / Y}(\beta)$ by repeating the set $\beta\left(\left[y_{0}\right]\right)$ once for every $y \in Y$, and the natural map is the map of coverings sending each of these copies to $\beta\left(\left[y_{0}\right]\right)$.

\section{Homotopy Invariance of Čech Homology.}

We wish to demonstrate that the inclusion $i: X \times 0 \hookrightarrow X \times[0,1]$ induces an equivalence $\breve{h}(X ; T) \rightarrow \breve{h}(X \times[0,1] ; T)$ for any homology theory $T$. Lemma 4.6 shows that the conditions of Proposition 2.4 are satisfied for the functor $\mathrm{RC}(i): \mathrm{RC}(X \times I) \rightarrow \mathrm{RC}(X \times 0)$, hence

$$
\text { holim }_{R C(X \times 0)} T \circ \Phi_{X \times 0} \rightarrow \operatorname{holim}_{R C(X \times I)} T \circ \Phi_{X \times 0} \circ R C(i)
$$

is a homotopy equivalence, so showing $\breve{h}(X ; T) \rightarrow \breve{h}(X \times[0,1] ; T)$ is a homotopy equivalence is equivalent to the assertion that the natural transformation $\Phi_{X \times 0} \circ R C(i) \rightarrow \Phi_{X \times[0,1]}$ induces a homotopy equivalence

$$
\operatorname{holim}_{R C(X \times I)} T \circ \Phi_{X \times 0} \circ R C(i) \rightarrow \operatorname{holim}_{R C(X \times I)} T \circ \Phi_{X \times[0,1]} .
$$

Notice that since $T$ is homotopy invariant $T$ applied to a weak homotopy equivalence will be a homotopy equivalence.

We will first establish some preliminaries.

Proposition 6.1. Let $\mathscr{U}=\left\{U_{\alpha}\right\}_{\alpha \in A}$ and $\mathscr{V}=\left\{V_{\beta}\right\}_{\beta \in B}$ be open coverings of spaces $X$ and $Y$, respectively, and let $\mathscr{U} \times \mathscr{V}$ be the open covering $\left\{U_{\alpha} \times V_{\beta}\right\}_{(\alpha, \beta) \in A \times B}$ of $X \times Y$. Then $N .(\mathscr{U} \times \mathscr{V})$ is naturally equivalent to $N . \mathscr{U} \times N . \mathscr{V}$.

Also, for any $n$ and $\epsilon>0$, with $\epsilon<\frac{1}{2^{n+1}}$, let $\mathscr{V}_{n, \epsilon}$ denote the open covering of $[0,1]$ given by $\left\{\left(\frac{k}{2^{n}}-\epsilon, \frac{k+1}{2^{n}}+\epsilon\right)\right\}_{k=0,1, \ldots, 2^{n}-1}$. Of course, $\left(-\epsilon, \frac{1}{2^{n}}+\epsilon\right)$ and $\left(1-\frac{1}{2^{n}}-\epsilon, 1+\epsilon\right)$ are to be interpreted as $\left[0, \frac{1}{2^{n}}+\epsilon\right)$ and $\left(1-\frac{1}{2^{n}}-\epsilon, 1\right]$, respectively. Note that $N . \mathscr{V}_{n, \epsilon}$ is weakly contractible.

Proposition 6.2. Let $\beta$ be any rigid covering of $X \times[0,1]$ whose underlying covering is of the form $\mathscr{U} \times \mathscr{V}_{n, \epsilon}$, for some open covering $\mathscr{U}$ of $X$. Then $\Phi_{X \times 0} \circ R C(i)(\beta) \rightarrow \Phi_{X \times[0,1]}(\beta)$ is a weak equivalence.

Proof. Clear from the preceding proposition and the contractibility of $N . \mathscr{V}_{n, \epsilon}$ 
Lemma 6.3. Let $\beta$ be any rigid covering of a compact Hausdorff space $X$, and let $x \in X$. Then there is an open set $V$ containing $x$ so that for all $v \in V$, $V \subseteq \beta(v)$.

Proof. Let $\left\{U_{\alpha}\right\}_{\alpha \in A}$ be a non-redundant listing of all the open sets appearing as $\beta(x)$ for some $x \in X$, so $A$ is finite. Let $S(x) \subseteq A$, $S(x)=\left\{\alpha \in A \mid x \in \overline{\beta^{-1} U_{\alpha}}\right\}$. Choose $V$ to be any open set contained inside

$$
\bigcap_{\alpha \in S(x)} U_{\alpha} \cap \bigcap_{\beta \in A-S(x)}\left(X-\overline{\beta^{-1}\left(U_{\beta}\right)}\right)
$$

$\mathrm{V}$ clearly satisfies the requirements of the lemma.

Corollary 6.4. Let $X$ be a compact Hausdorff space, and let $Y=[0,1]$. Let $\beta$ be a rigid covering of $X \times Y$. Then there is a rigid covering $\beta^{*} \geq \beta$ so that the underlying covering $\beta^{*}$ is of the form $\mathscr{U} \times \mathscr{V}$, and so that $N . \mathscr{V}$ is contractible.

Proof. First, it is clear from 6.3 that there is a finite open covering $\mathscr{U}=\left\{U_{\alpha}\right\}_{\alpha \in A}$ so that for every $u \in U_{\alpha}, U_{\alpha} \subseteq \beta u$. Further, it is standard that there are open coverings $\mathscr{W}=\left\{W_{\alpha}\right\}_{\alpha \in A}$ and $\mathscr{V}=\left\{V_{\beta}\right\}_{\beta \in B}$ of $X$ and $Y$ respectively, so that $\mathscr{W} \times \mathscr{V}$ refines $\mathscr{U}$, and that $\mathscr{V}=\mathscr{V}_{n, \epsilon}$ for some $n$ and $\epsilon$. It is clear that we may assume that the coverings $\mathscr{W}$ and $\mathscr{V}$ admit no proper subcovers. Since $X$ and $Y$ are compact Hausdorff spaces, we can choose open subsets $W_{\alpha}^{\prime} \subseteq W_{\alpha}$ and $V_{\beta}^{\prime} \subseteq V_{\beta}$ so that $\overline{W_{\alpha}^{\prime}} \subseteq W_{\alpha}$ and $\overline{V_{\beta}^{\prime}} \subseteq V_{\beta}$, and so that $\mathscr{W}^{\prime}=\left\{W_{\alpha}^{\prime}\right\}_{\alpha \in A}$ and $\mathscr{V}^{\prime}=\left\{V_{\beta}^{\prime}\right\}_{\beta \in B}$ are also open coverings of $X$ and $Y$, respectively. We may take $\mathscr{V}$ to be $\mathscr{V}_{n, \epsilon}$ for some $n$ and $\epsilon$. For each point $(x, y) \in X \times Y$, choose some sets $W_{\alpha}^{\prime}$ and $V_{\beta}^{\prime}$ so that $x \in W_{\alpha}^{\prime}$ and $y \in V_{\beta}^{\prime}$, and set $\beta^{*}(x, y)=W_{\alpha} \times V_{\beta}$. It follows directly from the construction that $\beta^{*}$ refines $\beta$, and since $\mathscr{W}^{\prime}$ and $\mathscr{V}^{\prime}$ admit no proper subcovers, it is clear that the underlying open covering of $\beta^{*}$ is $\mathscr{W} \times \mathscr{V}$, and $N . \mathscr{V}$ is weakly contractible.

THEOREM 6.5. The inclusion

$$
\underset{R C(X \times I)}{\operatorname{holim}} T \circ \Phi_{X \times 0} \circ R C(i) \underset{R C(X \times I)}{\operatorname{holim}} T \circ \Phi_{X \times[0,1]}
$$

is a homotopy equivalence. Consequently, Čech homology has the homotopy invariance property for compact Hausdorff spaces.

ProOF. By the preceding corollary, it suffices to check the result on rigid coverings whose underlying open coverings are of the form $\mathscr{W} \times \mathscr{V}$, with $N . \mathscr{V}$ weakly contractible. The result now follows from Proposition 6.1 and Proposition 6.2. 


\section{The "Strong Wedge" Property.}

Let $\left\{X_{i}\right\}_{i=1}^{\infty}$ be a countable family of based topological spaces. The "strong wedge" of the $X_{i}^{\prime} s$, which we by abuse of notation shall denote $\bigvee_{i=1}^{\infty} X_{i}$, is the subspace of $\prod_{i=1}^{\infty} X_{i}$ consisting of points which have at most one coordinate away from the basepoint. There are projection maps $\check{h}\left(\bigvee_{i=1}^{\infty} X_{i} ; T\right) \rightarrow \check{h}\left(X_{i} ; T\right)$, and hence a map

$$
\phi: \check{h}\left(\bigvee_{i=1}^{\infty} X_{i} ; T\right) \rightarrow \prod_{i=1}^{\infty} \breve{h}\left(X_{i} ; T\right)
$$

We wish to show that if each of the $X_{i}$ 's is a compact Hausdorff space, then $\phi$ is an equivalence.

We record a preliminary result.

Proposition 7.1. Let

$$
C_{1} \subseteq C_{2} \subseteq \ldots \subseteq C_{n} \subseteq \ldots
$$

be an increasing sequence of categories, with $C_{\infty}=\bigcup_{n} C_{n}$. Let $F$ be a functor from $C_{\infty}$ to Kan complexes. Then holim $C_{\infty} F$ is naturally equivalent to $\operatorname{holim}_{n} C_{n} F \mid C_{n}$. Here, $n \rightarrow$ holim $_{C_{n}} F \mid C_{n}$ is a contravariant functor from the partially ordered set $\mathbf{N}$ of positive integers to Kan complexes.

Proof. Let $\Phi$ be the covariant functor from $\mathrm{N}$ to small categories, given by $\Phi(n)=C_{n}$. Then we have the "Grothendieck construction" [17] $\mathscr{N}=\mathrm{N} \prec \Phi$, and an evident functor $s: \mathscr{N} \rightarrow C_{\infty}$. It is readily checked that this functor satisfies the hypotheses of [3, Theorem XI.9.2], and hence the pullback map holim $C_{\infty} F \rightarrow \operatorname{holim}_{\mathcal{N}} F \circ s$ is an equivalence. However, $\operatorname{holim}_{\mathcal{N}} F \circ s$ is easily identified with $\operatorname{holim}_{n \in \mathbf{N}} \Phi(n) F \mid \Phi(n)$, which is the required result.

THeOREM 7.2. Let $\left\{X_{i}\right\}_{i=0}^{\infty}$ be a family of based compact Hausdorff spaces. Then the natural map

$$
\phi: \check{h}\left(\bigvee_{i=1}^{\infty} X_{i} ; T\right) \rightarrow \prod_{i=1}^{\infty} \breve{h}\left(X_{i} ; T\right)
$$

is an equivalence.

Proof. Let $Z=\bigvee_{i=1}^{\infty} X_{i}$, and let $Z_{j}$ be the subspace $\bigvee_{i=j}^{\infty} X_{i}$. We have the projection $p_{j}: Z \rightarrow Z / Z_{j} \cong \bigvee_{i=1}^{j} X_{i}$. Let $\mathrm{RC}_{j}(Z) \subseteq \mathrm{RC}(Z)$ denote the image of the functor $\mathrm{RC}\left(p_{j}\right)$, and let $\mathrm{RC}_{\infty}(Z)=\bigcup_{j=1}^{\infty} \mathrm{RC}_{j}(Z) \subseteq \mathrm{RC}(Z)$. We claim that the restriction map

$$
\check{h}(Z ; T) \cong \operatorname{holim}_{R C(Z)} T \circ \Phi_{Z} \rightarrow \operatorname{holim}_{R C_{\infty}(Z)} T \circ \Phi_{Z}
$$


is a weak equivalence. By 2.8 , it will suffice to show that for every $\beta \in \mathrm{RC}(Z)$, there is a $\beta^{\prime} \in \mathrm{RC}(Z)$ and a $\beta^{\prime \prime} \in \mathrm{RC}_{\infty}(Z)$, so that $\beta^{\prime} \geq \beta$, $\beta^{\prime} \geq \beta^{\prime \prime}$, and so that $\Phi_{Z}\left(\beta^{\prime} \geq \beta^{\prime \prime}\right)$ is a weak equivalence of simplicial sets. Let $*$ denote the basepoint in $Z$. Then $\beta(*)$ is an open set containing $*$. By the definition of the strong wedge, there is a $j$ so that $Z_{j} \subseteq \beta(*)$. Choose an open set $V$ with $Z_{j} \subseteq V \subseteq \bar{V} \subseteq \beta(*)$; this is possible since $Z$ is compact Hausdorff. $\{Z-\bar{V}, \beta(*)\}$ is now an open covering of $Z$. By 4.4 , we may choose $\gamma \in \operatorname{RC}(Z)$ so that $\gamma(z)$ is equal to $Z-\bar{V}$ or $\beta(*)$ for all $z \in Z$. Note that for $z \in Z_{j}, \gamma(z)=\beta(*)$. Let $\beta^{\prime}=\beta \times_{X} \gamma$. It is clear that if $\beta^{\prime}(z) \cap Z_{j} \neq \emptyset$, then $\beta^{\prime}(z) \subseteq \beta(*)=\beta^{\prime}(*)$. Let $\beta^{\prime \prime}$ be defined by $\beta^{\prime \prime}(z)=\beta^{\prime}(z)$ if $\beta^{\prime}(z) \cap Z_{j}=\emptyset$, and $\beta^{\prime \prime}(z)=\beta(*)$ if $\beta^{\prime}(z) \cap Z_{j} \neq \emptyset$. Define a function $\theta$ from $Z$ to itself by letting $\theta(z)=*$ if $\beta^{\prime}(z) \cap Z_{j} \neq \emptyset$ and $\theta(z)=z$ otherwise. It is clear that $\beta^{\prime \prime}(z) \subseteq \beta^{\prime}(\theta(z)$ ), and so $\theta$ is in fact a map of coverings (indexed by $Z$ ). It therefore induces a map $R$. $\Sigma_{\beta^{\prime \prime}} \rightarrow R$. $\Sigma_{\beta^{\prime}}$. In view of 3.2 , this is a weak equivalence of simplicial sets. This shows that the map $\operatorname{holim}_{R C(Z)} T \circ$ $\Phi_{Z} \rightarrow \operatorname{holim}_{\mathrm{RC}_{\infty}(\mathrm{Z})} \mathrm{T} \circ \Phi_{Z}$ is an equivalence. But 7.1 now shows that $\operatorname{holim}_{R C_{\infty}(Z)} T \circ \Phi_{Z}$ is canonically equivalent to $\operatorname{holim}_{j} \mathrm{RC}_{j}(Z) T \circ \Phi_{Z}$. But it is easy to check that $T \circ \Phi_{Z} \mid R C_{j}(Z)$ is weakly equivalent to $T \circ \Phi_{Z / Z_{j}}$, and hence that $\operatorname{holim}_{R C_{j}(Z)} T \circ \Phi_{Z} \simeq \breve{h}\left(Z / Z_{j} ; T\right)$. On the other hand, the excision theorem 5.1 shows that $\breve{h}\left(Z / Z_{j} ; T\right) \simeq \prod_{i=1}^{j-1} \breve{h}\left(Z_{i} ; T\right)$. It follows that

$$
{ }_{R C_{\infty}(Z)} T \circ \Phi_{Z} \simeq \underset{j}{\operatorname{holim}} \prod_{i=1}^{j-1} \check{h}\left(Z_{i} ; T\right) \simeq \prod_{i=1}^{\infty} \check{h}\left(Z_{i} ; T\right)
$$

which is the required result.

\section{Bornologies, Compactifications, and Group Actions.}

A "space" will mean a locally compact Hausdorff space. If $X$ is a set, and $B_{1}$ and $B_{2}$ are subsets of $X \times X$, we write $B_{1} \circ B_{2}=\left\{\left(x_{1}, x_{2}\right) \in X \times X \mid \exists x^{\prime} \in X\right.$, with $\left(x, x^{\prime}\right) \in B_{1}$ and $\left.\left(x^{\prime}, x_{2}\right) \in B_{2}\right\}$. Also, let $B_{1}^{o p}=\left\{\left(x_{1}, x_{2}\right) \mid\left(x_{2}, x_{1}\right) \in B_{1}\right\}$.

Definition 8.1. A bornology on a space $X$ is a family $\mathscr{B}$ of subsets of $X \times X$, satisfying the following four conditions.

1. $B \in \mathscr{B} \Rightarrow \Delta \subseteq \mathscr{B}$, where $\Delta$ denotes the diagonal in $X \times X$.

2. $B \in \mathscr{B}$ and $\Delta \subseteq B^{\prime} \subseteq B \Rightarrow B^{\prime} \in \mathscr{B}$.

3. $B_{1}, B_{2} \in \mathscr{B} \Rightarrow B_{1} \circ B_{2} \in \mathscr{B}$.

4. $B \in \mathscr{B} \Rightarrow B^{o p} \in \mathscr{B}$.

A space equipped with a bornology will be referred to as a bornological space.

To get a category we need to specify the maps. 
Definition 8.2. Let $\left(X_{1}, \mathscr{B}_{1}\right)$ and $\left(X_{2}, \mathscr{B}_{2}\right)$ be bornological spaces, and let $f: X_{1} \rightarrow X_{2}$ be a (perhaps non-continuous) map. We say $f$ is bornological if it satisfies the following conditions:

1 For every $B \in \mathscr{B}_{1}, f \times f(B) \in \mathscr{B}_{2}$.

2 For every compact subset $K \subset X_{2}, \overline{f^{-1}(K)}$ is compact.

The associated category of bornological spaces will be denoted by Born.

Example 8.3. The metric bornology: An important example of a bornological space occurs when $X$ is a metric space. If $X$ is a metric space with metric $d$, we define a bornology $\mathscr{B}(d)$ on $X$ to consist of all sets $B \subseteq X \times X$, containing $\Delta$, and so that there is a real number $R$ so that $d\left(x_{1}, x_{2}\right) \leq R$ for all $\left(x_{1}, x_{2}\right) \in B$.

Definition 8.4. Let $(X, \mathscr{B})$ be a bornological space. If $A \subset X$ and $B \in \mathscr{B}$, we define $B[A]=\left\{x^{\prime} \in X \mid \exists x \in A:\left(x, x^{\prime}\right) \in B\right\}$. We shall write $B[x]$ for $B[\{x\}]$.

EXAMPLE 8.5. The continuously controlled bornology: Let $X$ be a space and let $i: X \rightarrow \bar{X}$ be an inclusion into a compact space. $i$ is assumed to be a homeomorphism onto its image. Let $\partial X=\bar{X}-X$. We define the continuously controlled bornology $\mathscr{B}(\bar{X}, \partial X)$ on $X$ as follows:

$B \in \mathscr{B}(\bar{X}, \partial X)$ if and only if the following holds: For every $y \in \partial X$ and for every open neighborhood $U$ of $y$ in $\bar{X}$ there exists an open neighborhood $V$ of $y$ in $\bar{X}$ so that $B[x] \subset U$ for all $x \in V \cap X$.

Definition 8.6. Let $X$ be a space with two bornologies $\mathscr{B}_{1}$ and $\mathscr{B}_{2}$. We shall say that $\mathscr{B}_{1}$ is finer than $\mathscr{B}_{2}$ or equivalently $\mathscr{B}_{2}$ is coarser than $\mathscr{B}_{1}$ if $\mathscr{B}_{1} \subset \mathscr{B}_{2}$ i. e. if the identity map from $\left(X, \mathscr{B}_{1}\right)$ to $\left(X, \mathscr{B}_{2}\right)$ is a bornological map.

Definition 8.7. If $X$ be is a bornological space imbedded as above, $X$ thus has an abstract bornology $\mathscr{B}$ and a continuously controlled bornology coming from the embedding. We say $\mathscr{B}$ is "small at $\infty$ " (relative to $i$ ) if $\mathscr{B}$ is finer than $\mathscr{B}(\bar{X}, \partial X)$ i.e. if for each $B \in \mathscr{B}, z \in \partial X$, and open set $U$ of $\bar{X}$ containing $z$, there is an open set $V$ of $\bar{X}$, with $z \in V \subseteq U$, so that for every $x \in V \cap X, B[x] \subseteq U$.

REMARK 8.8. There are many important examples of metric bornologies with compactifications that are small at infinity

1. When $X$ is a simply connected smooth manifold equipped with a complete Riemannian metric $d$ of nonpositive sectional curvature, then $X$ can be compactified by rays, and the bornology $\mathscr{B}(d)$ is small at $\infty$.

2. If $X=G / K$, where $G$ is a Lie group and $K$ its maximal compact subgroup, then $G / K$ is diffeomorphic to $R^{N}$ for $N=\operatorname{dim}(G / K)$, and can be 
compactified by adding an $S^{N-1}$ at $\infty$ so that any left $G$ - invariant Riemannian metric is small at $\infty$.

3. If $X$ is the universal cover of a finite simplicial graph, then $X$ can be compactified by adding a Cantor set at $\infty$, and the bornology associated to the simplicial metric on $X$ is small at $\infty$.

4. When $\Gamma$ is a torsion free word hyperbolic group in the sense of Gromov (see [11]), $\Gamma$ acts freely on a finite dimensional polyhedron $P_{d}(\Gamma)$ which can be compactified by adding its "hyperbolic boundary", as in [11]. The polyhedron is equipped with a metric, whose bornology is small at $\infty$.

Suppose now that $X$ is a space, equipped with a properly discontinuous left action by a group $\Gamma$, and suppose further that the action is "cocompact", i.e. $X / \Gamma$ is a compact Hausdorff space. $\Gamma$ also acts on $X \times X$ via the formula $\gamma \cdot\left(x, x^{\prime}\right)=\left(\gamma x, \gamma x^{\prime}\right)$. We say a subset $Z \subseteq X \times X$ is $\Gamma$-compact if $Z / \Gamma \subseteq X \times X / \Gamma$ is a compact set; note that $\Delta_{X} \subseteq X \times X$ is $\Gamma$-compact, since $X / \Gamma$ is compact. We now define a family $\mathscr{B}^{\Gamma}$ of subsets of $X \times X$ by declaring $B \subseteq X \times X$ is an element of $\mathscr{B}^{\Gamma}$ if $\Delta_{X} \subseteq B$ and $B$ is contained in a $\Gamma$-compact subset of $X \times X$.

Proposition 8.9. Suppose $\Gamma$ acts freely on $X$. Then $\mathscr{B}^{\Gamma}$ is a bornology on $X$.

Proof. Only condition 3 requires verification. Let $\pi_{1}, \pi_{2}: X \times X / \Gamma \rightarrow X / \Gamma$ be given by $\pi_{1}\left(\left[x, x^{\prime}\right]\right)=[x]$ and $\pi_{2}\left(\left[x, x^{\prime}\right]\right)=\left[x^{\prime}\right]$, respectively. Let $Z \subseteq(X \times X / \Gamma) \times(X \times X / \Gamma)$ be given by $Z=\left\{(\alpha, \beta) \mid \pi_{2} \alpha=\pi_{1} \beta\right\}$. There is a continuous map $\theta: Z \rightarrow X \times X / \Gamma$ given by $\theta\left(\left[x, x^{\prime}\right],\left[x^{\prime \prime}, x^{\prime \prime \prime}\right]\right)=\left[x, \gamma x^{\prime \prime \prime}\right]$, where $\gamma$ is the unique element of $\Gamma$ so that $\gamma x^{\prime \prime}=x^{\prime}$. Let $B_{1}, B_{2} \in \mathscr{B}$, and let $\bar{B}_{1}$ and $\bar{B}_{2}$ be $\Gamma$-invariant elements of $\mathscr{B} \Gamma$, so that $\bar{B}_{i} / \Gamma \subseteq X \times X / \Gamma$ is compact for each $i$, and so that $\bar{B}_{i} \supseteq B_{i}$ for each $i$. It is easy to see that such $\bar{B}_{i}$ 's exist. Now, $p\left(B_{1} \circ B_{2}\right) \subseteq p\left(\bar{B}_{1} \circ \bar{B}_{2}\right)=\theta\left(Z \cap\left(p \bar{B}_{1} \times p \bar{B}_{2}\right)\right)$, and $Z \cap\left(p \bar{B}_{1} \times p \bar{B}_{2}\right)$ is a closed subset of the compact set $p \bar{B}_{1} \times p \bar{B}_{2}$, and so $\theta\left(Z \cap\left(p \bar{B}_{1} \times p \bar{B}_{2}\right)\right)$ is compact. Consequently, $B_{1} \circ B_{2}$ is contained in a $\Gamma$-compact set, which is the result.

Definition 8.10. If in this situation $X$ is compactified to $\bar{X}$, we say that the group action is small at infinity if $\mathscr{B}^{\Gamma}$ is small at infinity

Definition 8.11. If $\left(X, \mathscr{B}_{X}\right)$ and $\left(Y, \mathscr{B}_{Y}\right)$ are bornological spaces we define the product bornology $\mathscr{B}_{X \times Y}$ on $X \times Y$ to consist of those subsets of

$$
(X \times Y) \times(X \times Y) \cong(X \times X) \times(Y \times Y)
$$

which contain the diagonal and are contained in subsets of the form $B_{1} \times B_{2} \subseteq X \times X \times Y \times Y \cong(X \times Y) \times(X \times Y)$, where $\quad B_{1} \in \mathscr{B}_{X} \quad$ and $B_{2} \in \mathscr{B}_{Y}$. 
Definition 8.12. If $Y$ is a bornological space with bornology $\mathscr{B}$ and $f: X \rightarrow Y$ we define the pullback bornology on $X$ to be

$$
f^{*}(\mathscr{B})=\left\{(f \times f)^{-1}(B) \mid B \in \mathscr{B}\right\} .
$$

In the following let $(\bar{X}, \partial X)$ be a pair of Hausdorff spaces, $\partial X$ closed in $\bar{X}$ and $\bar{X}-\partial X$ dense in $\bar{X}$. Let $U$ be a subset of $\partial X$. We define the bornology $\mathscr{B}_{U}$ of control with respect to $U$ as follows:

Definition 8.13. $B \in \mathscr{B}_{U}$ if and only if for every closed subset $Z$ of $\bar{X}$ with $Z \cap \partial X \subset U$ we have $\overline{B[Z]} \cap \partial X \subset U$.

Said with words $\mathscr{B}_{U}$ has to satisfy that if a closed set intersecting $\partial X$ inside $U$ is expanded by an element $B \in \mathscr{B}_{U}$ then the closure of the expansion also intersects $\partial X$ inside $U$.

Definition 8.14. If $A=\left\{U_{\alpha}\right\}$ is a collection of subsets of $\partial X$ then we define

$$
\mathscr{B}_{A}=\cap_{U \in A} \mathscr{B}_{U}
$$

REMARK 8.15. It is easy to see that continuous control is control with respect to the collection of all open subsets of $\partial X$. Hence the continuously controlled bornology is finer than the bornology defined by any collection of open sets.

Definition 8.16. Assume $X$ is a bornological space compactified to $\bar{X}$. A subset $Z$ of $\partial X$ is bornologically saturated with respect to a given bornology if the bornology is finer than $\mathscr{B}_{Z}$. In case $X$ is a metric space we say the set is boundedly saturated. In this case a closed subset of $X$ with closure meeting $\partial X$ inside $Z$ still meets $\partial X$ inside $Z$ after being expanded a bounded amount.

Remark 17. Given a collection of boundedly saturated sets $A=\left\{U_{\alpha}\right\}$ we clearly get a map from the metric bornology to $\mathscr{B}_{A}$. This will be used in the final section where we prove assembly map splittings.

\section{Bounded $K$-theory and $K$-theory with Continuous Control at $\infty$.}

Let $(X, \mathscr{B})$ be a bornological space, and let $R$ be a ring. By an $X$-labeled $R$ module, we mean a based free $R$-module $(F, \Phi)$, embedded as a submodule of the free $R$-module with basis $X \times N$. We get a corresponding "labeling function" $\phi: \Phi \rightarrow X$, and require that for every compact subset $K \subset X$, $\phi^{-1}(K)$ is finite. If $\left(F_{1}, \Phi_{1}, \phi_{1}\right)$ and $\left(F_{2}, \Phi_{2}, \phi_{2}\right)$ are $X$-labeled $R$-modules, then a linear transformation $f: F_{1} \rightarrow F_{2}$ is said to be bounded with respect to $\mathscr{B}$ if there is an element $B \in \mathscr{B}$ so that for any $\theta \in \Phi_{1}, f(\theta) \in\left(\phi_{2}^{-1}\left(B\left[\phi_{1} x\right]\right)\right)$. As in [15], the category of $X$-labeled $R$-modules and bounded linear transforma- 
tions is symmetric monoidal, being an additive category. We shall denote this category by $\mathscr{C}(X, \mathscr{B} ; R)$. The subcategory of isomorphisms is also symmetric monoidal and has an associated spectrum $((\mathscr{C}(X, \mathscr{B}, R)))$ will be denoted by $K(X, \mathscr{B}, R)$. Note that if $\mathscr{B}$ is the bornology associated to a metric, then $\mathscr{C}(X, \mathscr{B} ; R)$ coincides with the bounded category $\mathscr{C}(X ; R)$ of [15] applied to the metric space $X$. If $\mathscr{B}(\bar{X}, \partial X)$ is the continuously controlled bornology associated to a compactification $\bar{X}$ of $X$ then $\mathscr{C}(X, \mathscr{B}(\bar{X}, \partial X) ; R)$ is the continuously controlled category $\mathscr{B}(\bar{X}, \partial X ; R)$ of [2] and [5]. For brevity we shall continue to use the notation $\mathscr{B}(\bar{X}, \partial X ; R)$ for $\mathscr{C}(X, \mathscr{B}(\bar{X}, \partial X) ; R)$.

Let $\mathscr{B}_{E}$ denote the bornology associated to the standard Euclidean metric on $E$. Let $\mathscr{B}_{E^{n}}$ denote the $n$-fold product bornology $\mathscr{B}_{E \times \ldots \times E}$, described above. Then as in [4] or [5], we obtain a directed system

$$
\begin{aligned}
K\left(X, \mathscr{B}_{X}, R\right) \quad & \rightarrow \Omega K\left(X \times E, \mathscr{B}_{X \times E}, R\right) \rightarrow \cdots \\
\cdots & \rightarrow \Omega^{n} K\left(X \times E^{n}, \mathscr{B}_{X \times E^{n}}, R\right) \rightarrow \cdots
\end{aligned}
$$

of spectra, and $K\left(X \times E^{n}, \mathscr{B}_{X \times E^{n}}, R\right)$ is a component of $\Omega K\left(X \times E^{n+1}, \mathscr{B}_{X \times E^{n+1}}, R\right)$, so increasing $n$ introduces new negative homotopy groups, but keeps the homotopy groups in positive degrees. The homotopy colimit of this system will be written $K^{-\infty}(X, \mathscr{B}, R)$,

We briefly examine the functoriality properties of this construction. Consider a map $\left(X_{1}, \mathscr{B}_{1}\right) \stackrel{f}{\rightarrow}\left(X_{2}, \mathscr{B}_{2}\right)$ of bornological spaces.

Proposition 9.1. The constructions $K(X, \mathscr{B}, R)$ and $K^{-\infty}(X, \mathscr{B}, R)$ are functorial for maps of bornological spaces.

Proof. Clear from the definitions.

We now examine the functoriality of these constructions, when a bornology is small at infinity.

Definition 9.2. Let $(\bar{X}, \partial X)$ and $\left(\overline{X^{\prime}}, \partial X^{\prime}\right)$ be pairs of locally compact Hausdorff spaces. $X=\bar{X}-\partial X$ and $X^{\prime}=\overline{X^{\prime}}-\partial X^{\prime}$. A set map $f:(\bar{X}, \partial X) \rightarrow\left(\overline{X^{\prime}}, \partial X^{\prime}\right)$ is eventually continuous if the following conditions hold.

1. $f(X) \subseteq f\left(X^{\prime}\right)$.

2. If $K$ is a compact subset of $X^{\prime}$, then the closure of $f^{-1}(K)$ in $X$ is compact.

3. $f$ is continuous at points of $\partial X$.

We denote the category with objects compact Hausdorff pairs and morphisms eventually continuous maps by $\mathscr{C E}$

Now suppose that we are given a bornological space $(X, \mathscr{B})$, and an inclusion $i: X \rightarrow \bar{X}$, where $\bar{X}$ is compact. $\partial X$ will denote $\bar{X}-X$, and $i$ is assumed to be a homeomorphism onto its image. Then it is clear that the object sets of $\mathscr{C}(X, \mathscr{B} ; R)$ and $\mathscr{C}(X, \mathscr{B}(\bar{X}, \partial X ; R))=\mathscr{B}(\bar{X}, \partial X ; R)$ are identical. 
If we further assume that the bornology $\mathscr{B}$ is small at $\infty$, then it is also clear from the definitions that if a linear transformation of $X$-labeled $R$-modules is bounded with respect to $\mathscr{B}$, then that same linear transformation is controlled at all points of $\partial X$. The conclusion is that we have a symmetric monoidal functor $\mathscr{C}(X, \mathscr{B} ; R) \rightarrow \mathscr{B}(\bar{X}, \partial X ; R)$, and hence maps of spectra $K(X, \mathscr{B}, R) \rightarrow K(\bar{X}, \partial X, R)$ and $K^{-\infty}(X, Y, R) \rightarrow K^{-\infty}(\bar{X}, \partial X ; R)$. This map turns out to be a natural transformation of functors on an appropriate category, which we now describe. The objects are triples $(\bar{X}, \partial X, \mathscr{B})$, where $\bar{X}$ is a compact space, $\partial X \subseteq \bar{X}$ is a closed subspace, and $\mathscr{B}$ is a bornology on $X=\bar{X}-\partial X$, which is small at $\infty$ relative to the inclusion $X \rightarrow \bar{X}$. A morphism in the category from $(\bar{X}, \partial X, \mathscr{B})$ to $\left(\overline{X^{\prime}}, \partial X^{\prime}, \mathscr{B}^{\prime}\right)$ is a set map (of pairs) $(\bar{X}, \partial X) f\left(\overline{X^{\prime}}, \partial X^{\prime}\right)$ which is eventually continuous and so that $f \mid X$ is bornological. We call this category $\mathscr{\mathscr { P }}$. We say an object $(\bar{X}, \partial X, \mathscr{B})$ of $\mathscr{\mathscr { P }}$ is finite if $\partial X=\emptyset$ and $\mathscr{B}$ consists of all subsets of $X \times X$.

Proposition 9.3. The maps of spectra

$$
K(X, \mathscr{B} ; R) \rightarrow K(\bar{X}, \partial X ; R)
$$

and

$$
K^{-\infty}(X, \mathscr{B} ; R) \rightarrow K^{-\infty}(\bar{X}, \partial X ; R)
$$

form natural transformations of functors on $\mathscr{S}$. We refer to the natural transformation as $\eta . \eta(\bar{X}, \partial X, \mathscr{B})$ is a weak equivalence of spectra when $(\bar{X}, \partial X, \mathscr{B})$ is finite.

Proof. The naturality is evident. When $(\bar{X}, \partial X, \mathscr{B})$ is finite, both functors clearly take the value $K(R)$ and $K^{-\infty}(R)$ respectively and the map $\eta$ is clearly an equivalence.

\section{0. $K$-theory with Continuous Control at Infinity.}

We shall examine the excision properties of the continuously controlled construction.

As usual, let $(\bar{X}, \partial X)$ be a pair of spaces, with $\bar{X}$ compact Hausdorff and $\partial X$ closed. For any set $Z \subseteq \bar{X}$, let the accumulation set of $Z, \mathscr{A}(Z)$, be the set of all points $x \in \bar{X}$ so that every open neighborhood of $x$ contains infinitely many points of $Z$. Note that if $Z$ has the property that every compact subset of $\bar{X}$ contains only finitely many points of $Z$, then $\mathscr{A}(Z)$ is contained in $\partial X$. As in [5], for any subset $U \subseteq \partial X$, let $\mathscr{B}(\bar{X}, \partial X ; R)_{U}$ denote the full subcategory on $\mathscr{B}(\bar{X}, \partial X ; R)$ on the objects $(F, B, \phi)$ for which $\mathscr{A}(\phi(B)) \subseteq U$. We denote the corresponding $K$-theory spectrum by $K(\bar{X}, \partial X ; R)_{U}$, and also construct, the spectrum $K^{-\infty}(\bar{X}, \partial X ; R)_{U}$ by giving 
$X \times E^{n}$ the product bornology and consider objects with accumulation points in $\partial X \times E^{n}$ contained in $U \times E^{n}$. If $V \subseteq U$, we also define $\mathscr{B}(\bar{X}, \partial X ; R)_{U}^{V}$ to be the category whose objects are the same as those of $\mathscr{B}(\bar{X}, \partial X ; R)_{U}$, but where two morphisms $f$ and $g$ from $\left(F_{1}, B_{1}, \phi_{1}\right)$ to $\left(F_{2}, B_{2}, \phi_{2}\right)$ in $\mathscr{B}(\bar{X}, \partial X ; R)_{U}$ are identified if there is a neighborhood $W$ of $V$ so that $f(b)=g(b)$ for all $b \in B_{1}$ such that $\phi(b) \in W$. We get corresponding $K$-theory spectra $K(\bar{X}, \partial X ; R)_{U}^{V}$ and $K^{-\infty}(\bar{X}, \partial X ; R)_{U}^{V}$. The key result is now as follows.

Proposition 10.1. Let $C \subseteq D$ be closed subsets of $\partial X$. Then there is a sequence of maps of spectra

$$
K^{-\infty}(\bar{X}, \partial X ; R)_{C} \rightarrow K^{-\infty}(\bar{X}, \partial X ; R)_{D} \rightarrow K^{-\infty}(\bar{X}, \partial X ; R)_{D}^{D-C}
$$

which is a fibration up to homotopy.

Proof. For the case $D=\partial X$, this is [5, Corollary 1.30]. The proof for a general $D$ is identical.

REMARK 10.2. This is the key excision result. In $L$-theory a result of this type is proved in [5, Lemma 5.2]. In $A$-theory this is proved in [7, Proposition 2.12], and in topological $K$-theory this is proved in [12, Proposition 9.2]. Given an excision result of this type it is formal to generalize the rest of the methods in this paper to these other theories.

Definition 10.3. Let $C_{1}$ and $C_{2}$ be two closed subsets of $\partial X$. We say the pair $\left(C_{1}, C_{2}\right)$ is excisive if we can find an open set $V$ in $X$ so that $C_{2}-C_{1} \subseteq V$ and $\bar{V} \cap C_{1} \subseteq C_{2}$. For two arbitrary subsets $U_{1}$ and $U_{2}$ of $\partial X$ we say the pair $\left(U_{1}, U_{2}\right)$ is excisive if every compact subset $C$ of $U_{1} \cup U_{2}$ is contained in $C_{1} \cup C_{2}$ where $\left(C_{1}, C_{2}\right)$ is an excisive pair of closed subsets with $C_{i} \subset U_{i}$.

Recall a set $C \subseteq X$ is called functionally closed if there is a continuous function $f: X \rightarrow[0,1]$ with $C=f^{-1}(0)$.

Lemma 10.4. Assume $C_{i} \subset \partial X \subset X$ and $C_{i}$ are functionally closed in $X$. Then $\left(C_{1}, C_{2}\right)$ is an excisive pair.

Proof. Choose functions $f_{i}$ so that $C_{i}=f_{i}^{-1}(0)$. Put $V=\left\{x \mid f_{2}(x)<f_{1}(x)\right\}$

Proposition 10.5. Let $\bar{X}$ be a compact Hausdorff space, and let $\partial X \subseteq X$ be closed. Then any pair of open subsets $U_{1}, U_{2} \subseteq \partial X$ is excisive.

Proof. Let $C \subseteq U_{1} \cup U_{2}$. Consider the closed subset $C-U_{2} \cap C$ of $\partial X$. $C-U_{2} \cap C \subseteq U_{1}$, and it is a standard fact from point set topology that there is an open subset $V_{1}$ of $\partial X$, with $C-U_{2} \cap C \subseteq V_{1}$ and $\overline{V_{1}} \subseteq U_{1}$. Note that $C \subseteq V_{1} \cup U_{2}$. Now consider the closed subset $C-V_{1} \cap C$ of $\partial X$. As before, 
we choose an open subset $V_{2}$ of $\bar{X}$, with $C-V_{1} \cap C \subseteq V_{2}$, and so that $\overline{V_{2}} \subseteq U_{2}$. Now let $C_{i}=C \cap \overline{V_{i}}$. These $C_{i}$ may not form an excisive pair, but using the Tietze extension theorem they may be enlarged to functionally closed sets hence satisfying the conditions.

Lemma 10.6. If $C_{i} \subseteq \partial X$ are closed subsets and $\left(C_{1}, C_{2}\right)$ is an excisive pair, then

$$
K^{-\infty}(\bar{X}, \partial X ; R)_{C_{2}}^{C_{2}-C_{1} \cap C_{2}} \rightarrow K^{-\infty}(\bar{X}, \partial X ; R)_{C_{1} \cup C_{2}}^{C_{1} \cup C_{2}-C_{1}}
$$

is an equivalence of spectra.

Proof. It will suffice to show that the map

$$
K(\bar{X}, \partial X ; R)_{C_{2}}^{C_{2}-C_{1} \cap C_{2}} \rightarrow K(\bar{X}, \partial X ; R)_{C_{1} \cup C_{2}}^{C_{1} \cup C_{2}-C_{1}}
$$

is an equivalence, and pass to direct limits over the directed system defining $K^{-\infty}$ by crossing everything with $E^{n}$. But by 2.3 , it will suffice to show that the map

$$
N . \mathscr{B}(\bar{X}, \partial X ; R)_{C_{2}}^{C_{2}-C_{1} \cap C_{2}} \rightarrow N . \mathscr{B}(\bar{X}, \partial X ; R)_{C_{1} \cup C_{2}}^{C_{1} \cup C_{2}-C_{1}}
$$

induced by the evident inclusion functor is an equivalence. Since all morphisms in both categories are isomorphisms, it will suffice to show that every object in $\mathscr{B}(\bar{X}, \partial X ; R)_{C_{1} \cup C_{2}}^{C_{1} \cup C_{2}-C_{1}}$ is isomorphic to an object of $\mathscr{B}(\bar{X}, \partial X ; R)_{C_{2}}^{C_{2}-C_{1} \cap C_{2}}$. Choose $V \subseteq \bar{X}$ an open set so that

$$
C_{2}-C_{1} \subseteq V \text { and } \bar{V} \cap C_{1} \subseteq C_{2}
$$

We now write $(F, B, \phi)$ as a direct sum $\left(F_{1}, B_{1}, \phi_{1}\right) \oplus\left(F_{2}, B_{2}, \phi_{2}\right)$, where $B_{1}=\phi^{-1}(X-V)$ and $B_{2}=\phi^{-1}(V)$. The inclusion $\left(F_{2}, B_{2}, \phi_{2}\right) \hookrightarrow(F, B, \phi)$ is an isomorphism in the category $\mathscr{B}(\bar{X}, \partial X ; R)_{C_{1} \cup C_{2}}^{C_{1}-C_{1}}$ with inverse the projection since the composite only differs from the identity on a submodule with support at infinity contained in $C_{1}$. But, $\left(F_{2}, B_{2}, \phi_{2}\right)$ is clearly an object in $\mathscr{B}(\bar{X}, \partial X ; R)_{C_{2}}^{C_{1} \cap C_{2}}$, since the support of $\left(F_{2}, B_{2}, \phi_{2}\right)$ is contained in $V$, and $\mathscr{A}\left(\phi_{2}\left(B_{2}\right)\right) \subseteq \bar{V} \cap\left(C_{1} \cup C_{2}\right) \subseteq C_{1}$. To finish off the proof we need to verify the existence of such an open set $V$. Let $f_{i}: \bar{X} \rightarrow[0,1]$ be a continuous functions so that $f_{i}^{-1}(0)=C_{i}$. We may then put $V=\left\{y \mid f_{2}(y)<f_{1}(y)\right\}$.

THeOREM 10.7. If $\left(U_{1}, U_{2}\right)$ is excisive, then there is a pushout diagram of spectra

$$
\begin{aligned}
& K^{-\infty}(\bar{X}, \partial X ; R)_{U_{1} \cap U_{2}} \rightarrow K^{-\infty}(\bar{X}, \partial X ; R)_{U_{2}} \\
& K^{-\infty}(\bar{X}, \partial X ; R)_{U_{1}} \rightarrow K^{-\infty}(\bar{X}, \partial X ; R)_{U_{1} \cup U_{2}}
\end{aligned}
$$


Proof. The category $\mathscr{B}(\bar{X}, \partial X ; R)_{U}$ is always the direct limit of the categories $\mathscr{B}(\bar{X}, \partial X ; R)_{C}$, where $C$ runs over the compact subsets of $U$, since the set $\mathscr{A}(\phi(B))$ is always a closed, hence compact, subset of $\partial X$. Given $C \subseteq U_{1} \cup U_{2}$, we can find $C_{i}$ so $C \subseteq C_{1} \cap C_{2}$ with $\left(C_{1}, C_{2}\right)$ an excisive pair. From 10.1 we get a diagram of fibrations

$$
\begin{gathered}
K^{-\infty}(\bar{X}, \partial X ; R)_{C_{1} \cap C_{2}} \rightarrow K^{-\infty}(\bar{X}, \partial X ; R)_{C_{2}} \rightarrow K^{-\infty}(\bar{X}, \partial X ; R)_{C_{2}}^{C_{2}-C_{1} \cap C_{2}} \\
\downarrow \\
\downarrow \\
K^{-\infty}(\bar{X}, \partial X ; R)_{C_{1}} \rightarrow K^{-\infty}(\bar{X}, \partial X ; R)_{C_{1} \cup C_{2}} \rightarrow K^{-\infty}(\bar{X}, \partial X ; R)_{C_{1} \cup C_{2}}^{C_{1} \cup C_{2}-C_{1}}
\end{gathered}
$$

The right hand vertical arrow is an equivalence of spectra by Lemma 10.6. This will give the result by taking a limit over compact $C_{i} \subset U_{i}$, using the excisiveness assumption.

Suppose $\left\{U_{\alpha}\right\}_{\alpha \in A}$ is any open covering of $\partial X$. Let $\mathscr{F}(A)$ denote the collection of nonempty subsets of $A$. This is a partially ordered set under inclusion. We think of $\mathscr{F}(A)$ as a category in the usual fashion, so there is a unique map from $S \rightarrow T$ if $T \subseteq S$. Let $\sigma\left\{\alpha_{1}, \ldots, \alpha_{s}\right\}=U_{\alpha_{1}} \cap \ldots \cap U_{\alpha_{s}} \subseteq \partial X . \quad$ Then $\quad S \rightarrow K(\bar{X}, \partial X ; R)_{\sigma(S)} \quad$ and $S \rightarrow K^{-\infty}(\bar{X}, \partial X, R)_{\sigma(S)}$ define functors $T$ and $\mathscr{T}$ from $\mathscr{F}(A)$ to the category of spectra. Further, if we let $E$ and $\mathscr{E}$ denote the constant functors with values $K(\bar{X}, \partial X ; R)$ and $K^{-\infty}(\bar{X}, \partial X ; R)$ respectively, we have natural transformations $T \rightarrow E$ and $\mathscr{T} \rightarrow \mathscr{E}$, giving maps

$$
\underset{\mathscr{F}(A)}{\operatorname{hocolim}} \quad T \rightarrow \underset{\mathscr{F}(A)}{\operatorname{hocolim}} \quad E
$$

and

$$
\underset{\mathscr{F}(A)}{\operatorname{hocolim}} \quad \mathscr{T} \rightarrow \underset{\mathscr{F}(A)}{\operatorname{hocolim}} \quad \mathscr{E} .
$$

Since $\mathscr{F}(A)$ is a left filtering category,$N . \mathscr{F}(A)$ is clearly weakly contractible as in [16]. The natural maps from homotopy colimits to colimits now give maps

and

$$
\underset{\mathscr{F}(A)}{\operatorname{hocolim}} \quad E \rightarrow K(\bar{X}, \partial X ; R)
$$

$$
\underset{\mathscr{F}(A)}{\operatorname{hocolim}} \quad \mathscr{E} \rightarrow K^{-\infty}(\bar{X}, \partial X ; R)
$$

and hence by composition maps

and

$$
\underset{\mathscr{F}(A)}{\operatorname{hocolim}} T \rightarrow K(\bar{X}, \partial X ; R)
$$




$$
\underset{\mathscr{F}(A)}{\operatorname{hocolim}} \mathscr{T} \rightarrow K^{-\infty}(\bar{X}, \partial X ; R)
$$

We wish to prove that the map

$$
\underset{\mathscr{F}(A)}{\operatorname{hocolim}} \mathscr{T} \rightarrow K^{-\infty}(\bar{X}, \partial X ; R)
$$

is a weak equivalence of spectra.

THEOREM 10.8. Let $A=\{1,2\}$, and suppose we have an open covering $\left\{U_{1}, U_{2}\right\}$ of $\partial X$. Then the map

is an equivalence of spectra.

$$
\underset{\mathscr{F}(A)}{\operatorname{hocolim}} \mathscr{T} \rightarrow K^{-\infty}(\bar{X}, \partial X ; R)
$$

Proof. In this case, the homotopy colimit hocolim $\operatorname{FF}_{\mathscr{F}(A)} \mathscr{T}$ is just the homotopy pushout of the diagram

$$
\begin{aligned}
& K^{-\infty}(\bar{X}, \partial X ; R)_{U_{1} \cap U_{2}} \rightarrow K^{-\infty}(\bar{X}, \partial X ; R)_{U_{2}} \\
& \quad \downarrow \\
& K^{-\infty}(\bar{X}, \partial X ; R)_{U_{1}}
\end{aligned}
$$

The result now follows from Theorem 10.7 and Proposition 10.5.

Corollary 10.9. Let $A$ be any finite set, and suppose we have an open covering $\left\{U_{\alpha}\right\}_{\alpha \in A}$ of $\partial X$. Then the natural map

is an equivalence.

$$
\underset{\mathscr{\mathscr { F }}(A)}{\operatorname{hocolim}} \mathscr{T} \rightarrow K^{-\infty}(\bar{X}, \partial X ; R)
$$

Proof. This follows in a straightforward fashion by repeated use of 10.8 .

Corollary 10.10. Suppose $A$ is any set, and $\left\{U_{\alpha}\right\}_{\alpha \in A}$ is an open covering so that only finitely many distinct sets occur among the $U_{\alpha}$ 's. Then the map

$$
\underset{\mathscr{F}(A)}{\operatorname{hocolim}} \mathscr{T} \rightarrow K^{-\infty}(\bar{X}, \partial X ; R)
$$

is an equivalence of spectra.

Proof. Let $A_{0} \subseteq A$ be any finite subset of $A$ so that for any $\alpha \in A$, there is an $\alpha_{0} \in A_{0}$ so that $U_{\alpha}=U_{\alpha_{0}}$. Then we have a commutative diagram

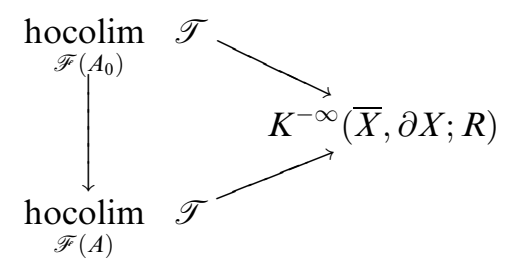


of spectra, and the diagonal arrow is an equivalence by 10.9. On the other hand, it follows directly from 3.1 that the vertical arrow is an equivalence. This gives the result.

Corollary 10.11. Let $\beta \in \mathrm{RC}(\partial X)$. The natural map

$$
\underset{S \in \mathscr{F}(\partial X)}{\operatorname{hocolim}} K^{-\infty}(\bar{X}, \partial X ; R)_{\sigma(S)} \rightarrow K^{-\infty}(\bar{X}, \partial X ; R)
$$

is an equivalence of spectra.

This does not actually need the rigidity of the coverings, but we shall only apply it in that case.

We now wish to construct a homotopy natural transformation from

$$
K^{-\infty}(\bar{X}, \partial X ; R) \rightarrow \Sigma \check{h}(\partial X ; R),
$$

as functors on $\mathscr{C E}$ (see definition 9.2). To do this, we will need to compare various constructions on the category $\mathscr{C E E}$. A convenient framework for discussing these is given by the following definition.

Definition 10.12 . We shall use $\mathscr{M}$ to denote the category whose objects are quadruples $(\bar{X}, \partial X, \beta, S)$, where $(\bar{X}, \partial X)$ is a compact Hausdorff pair, where $\beta \in R C(\partial X)$, and where $S$ is a finite subset of $\partial X$. A morphism from $\left(X_{1}, \partial X_{1}, \beta_{1}, S_{1}\right)$ to $\left(X_{2}, \partial X_{2}, \beta_{2}, S_{2}\right)$ is determined by a morphism $f:\left(X_{1}, \partial X_{1}\right) \rightarrow\left(X_{2}, \partial X_{2}\right)$ in $\mathscr{M}$ so that $\beta_{1}$ refines $R C\left(f \mid \partial X_{1}\right)\left(\beta_{2}\right)$, and so that $f\left(S_{1}\right) \subseteq S_{2}$. Any functor $F: \underline{M} \rightarrow \underline{\text { spectra }}$ determines a functor $\check{F}: \underline{\mathscr{E E}} \rightarrow \underline{\text { spectra }}$ on objects via the formula

$$
\check{F}(\bar{X}, \partial X)=\operatorname{holim}_{\beta \in R C(\partial X)} \underset{S \in \mathscr{F}(\partial X)}{\operatorname{hocolim}} F(\bar{X}, \partial X, \beta, S)
$$

and on morphisms via evident pullback and pushforward maps of homotopy limits and colimits, respectively. Natural transformations of functors on $\underline{M}$ determine natural transformations of functors on $\mathscr{C} E$, and weak equivalences determine weak equivalences.

Let $\mathscr{E}, \mathscr{T}, \mathscr{C}, \mathscr{D}$, and $\mathscr{D}^{0}$ be defined by the following formulae.

$$
\begin{gathered}
\mathscr{E}(\bar{X}, \partial X, \beta, S)=K^{-\infty}(\bar{X}, \partial X ; R) \\
\mathscr{T}(\bar{X}, \partial X, \beta, S)=K^{-\infty}(\bar{X}, \partial X ; R)_{\sigma_{\beta}(S)} \\
\mathscr{C}(\bar{X}, \partial X, \beta, S)= \begin{cases}K^{-\infty}(R) & \text { if } \sigma_{\beta}(S)=\emptyset \\
* & \text { if } \sigma_{\beta}(S) \neq \emptyset\end{cases}
\end{gathered}
$$




$$
\begin{aligned}
& \mathscr{D}(\bar{X}, \partial X, \beta, S)= \begin{cases}* & \text { if } \sigma_{\beta}(S)=\emptyset \\
K^{-\infty}(R) & \text { if } \sigma_{\beta}(S) \neq \emptyset\end{cases} \\
& \mathscr{D}^{0}(\bar{X}, \partial X, \beta, S)=K^{-\infty}(R)
\end{aligned}
$$

The effect on morphisms is defined in the evident way.

Proposition 10.13. The inclusions of spectra

$$
K^{-\infty}(\bar{X}, \partial X ; R)_{\sigma_{\beta}(S)} \subseteq K^{-\infty}(\bar{X}, \partial X ; R)
$$

induce an equivalence of functors $\check{\mathscr{T}} \rightarrow \check{\mathscr{E}}$, and $\check{\mathscr{E}}$ is weakly equivalent to the functor

$$
(\bar{X}, \partial X) \rightarrow K^{-\infty}(\bar{X}, \partial X ; R)
$$

on $\underline{\mathscr{C} E}$.

Proof. Follows directly from 10.11 .

Proposition 10.14. $\check{\mathscr{D}}$ is naturally equivalent to the functor

$$
(\bar{X}, \partial X) \rightarrow \breve{h}\left(\partial X_{+}, K^{-\infty}(R)\right)
$$

on $\underline{\mathscr{C} E}$. Similarly, $\check{\mathscr{D}}^{0}$ is weakly equivalent to the constant functor with value $K^{-\infty}(R)$.

Proof. If $\sigma_{\beta}(S)=\emptyset$ then the value of $\mathscr{D}(X, \partial X, \beta, T)$ is $*$ on any $T$ to the left of $S$, hence we do not change the homotopy colimit by restricting to the subcategory of $\mathscr{F}(\partial X)$ with $\sigma_{\beta}(S) \neq \emptyset$. Now use the standard fact that for a constant spectrum valued functor $\Phi$ with value $\mathscr{S}$ on a category $\underline{C}$, hocolim $_{\underline{C}} \Phi \cong N .(\underline{C})_{+} \wedge \mathscr{S}$. In our first case, $\mathscr{S}=K^{-\infty}(R)$ and $\underline{C}$ is the full subcategory of $\mathscr{F}(\partial X)$ consisting of $S$ so that $\sigma_{\beta}(S) \neq \emptyset$, hence $N$. $\underline{C}$ is precisely the nerve of the covering $\beta$. In the second case, $\underline{C}$ is the whole category $\mathscr{F}(\partial X)$.

COROLlary 10.15. $\check{\mathscr{C}}$ is naturally weakly equivalent to $\Sigma \check{h}\left(\partial X, K^{-\infty}(R)\right)$.

PROOF. We have a cofibration sequence of spectrum valued functors

$$
\mathscr{D} \rightarrow \mathscr{D}^{0} \rightarrow \mathscr{C}
$$

on $\underline{M}$. This gives a cofibration sequence

$$
\check{\mathscr{D}} \rightarrow \check{\mathscr{D}}^{0} \rightarrow \check{\mathscr{C}}
$$

on $\mathscr{C E E}$, which is the required result.

Finally, we define a natural transformation $\pi: \mathscr{T} \rightarrow \mathscr{C}$ by letting 


$$
\pi(\bar{X}, \partial X, \beta, S): K^{-\infty}(\bar{X}, \partial X ; R)_{\sigma_{\beta}(S)} \rightarrow K^{-\infty}(R)
$$

be induced by the forgetful functor when $\sigma_{\beta}(S)=\emptyset$, and letting

$$
\pi(\bar{X}, \partial X, \beta, S): K^{-\infty}(\bar{X}, \partial X ; R)_{\sigma_{\beta}(S)} \rightarrow *
$$

be the constant map when $\sigma_{\beta}(S) \neq \emptyset$. (Note that for any object $(F, B, \phi) \in \mathscr{B}(\bar{X}, \partial X ; R)_{\emptyset}, F$ is finitely generated. Indeed, the forgetful functor which takes $(F, B, \phi)$ to $F$ induces an equivalence of spectra. )

THEOREM 10.16. The diagram of functors

$$
\check{\mathscr{E}} \longleftarrow \check{\mathscr{T}} \rightarrow \check{\mathscr{C}} \longleftarrow \Sigma \check{h}\left(-, K^{-\infty}(R)\right)
$$

exhibits a homotopy natural transformation from the functor

$$
(\bar{X}, \partial X) \rightarrow K^{-\infty}(\bar{X}, \partial X ; R)
$$

to the functor

$$
(\bar{X}, \partial X) \rightarrow \Sigma \check{h}\left(\partial X, K^{-\infty}(R)\right),
$$

which we also refer to as $\pi$. The homotopy natural transformation $\pi$ is a weak equivalence for pairs of the form $(\bar{X}, \partial X)$, of compact metric spaces.

Proof. We need to show that $\pi$ induces an isomorphism on homotopy groups for $(\bar{X}, \partial X)$ a compact metrizable pair. It was shown in [5, Corollary 1.24] that $K^{-\infty}(\bar{X}, \partial X ; R)$ only depends on $\partial X$ when $\bar{X}$ is metrizable, and satisfies the Steenrod axioms [5, Theorem 1.36]. Since we have shown 10.11 and 5.1 that $\Sigma \grave{h}\left(\partial X ; K^{-\infty}(R)\right)$ satisfies the Steenrod axioms, and we clearly have an equivalence when $\partial X=\emptyset$, the result follows from [14].

\section{Variant Čech constructions.}

We occasionally need to consider Čech constructions where we do not allow coverings by all open sets. Given a family of open coverings $\mathscr{F}$ of a topological space $X$ which is closed under intersections in the sense that if $\left\{U_{\alpha}\right\}$ and $\left\{V_{\beta}\right\}$ belong to $\mathscr{F}$ then $\left\{U_{\alpha} \cap V_{\beta}\right\}$ does also belong to $\mathscr{F}$. We can then consider the subcategory of the category of regular coverings of $X$ consisting of regular coverings with image belonging to $\mathscr{F}$. This will be a partially ordered subset $\mathrm{RC} \mathscr{F}(X)$ of the partially ordered set of rigid coverings. We shall define $\breve{h}(X, \mathscr{F} ; T)$ by taking the holim over $\mathrm{RC} \mathscr{F}(X)$ rather than the full category of regular coverings. The functorial properties of $\breve{h}(X, \mathscr{F} ; T)$ are complicated to state, but if we have a group acting on $X$ and $\mathscr{F}$ is invariant under the group action, we do get an induced action on $\breve{h}(X, \mathscr{F} ; T)$. In case $X$ is a bornological space with bornology $\mathscr{B}$ compactified to $\bar{X}$ by 
adding $\partial X$ we define $\breve{h}(\partial X, \mathscr{B} ; T)$ by using the family of finite coverings by bornologically saturated open subsets of $\partial X$. The excision results above generalize to produce a homotopy natural transformation

$$
K^{-\infty}(X, \mathscr{B}) \rightarrow \check{h}\left(\partial X_{+}, \mathscr{B} ; K^{-\infty}\right) .
$$

We do not state the functorial properties of this construction but once again we do get induced actions if the bornology is invariant under a group action.

REMARK 11.1. Throughout the paper we have considered coverings by open sets. Many of the results will hold for more general kinds of coverings. The key point in constructing a homotopy natural transformation from a controlled theory to a Čech theory is excision, so as long as any pair of intersections of the sets in the covering is excisive we do get a homotopy natural transformation of spectra as above.

\section{Splitting assembly maps.}

The purpose of this section is to show the main theorem and variations. First we remove the metrizability condition of [5]. We do not know whether this adds any groups to the list of groups for which assembly maps split, but it is the easiest example to demonstrate our techniques. The following is a slightly more general statement than Theorem A

Theorem 12.1. Assume $\Gamma$ is a group with a finite $B \Gamma$ and that $E \Gamma$ has an equivariant compact Hausdorff compactification $\overline{E \Gamma}$ such that the $\breve{C}$ ech homology is trivial when using coefficients in the relevant spectrum $K^{-\infty}(R)$ or $\mathrm{L}^{-\infty}(\mathrm{R})$ and such that the action is small at infinity. Then

a) If $R$ is any ring then the assembly map

$$
B \Gamma_{+} \wedge K^{-\infty}(R) \rightarrow K^{-\infty}(R \Gamma)
$$

is equivalent to an inclusion of a direct summand of spectra.

b) If $R$ is a ring with involution such that $K_{-i}(R)=0$ for i sufficiently large then the assembly map

$$
B \Gamma_{+} \wedge \mathrm{L}^{-\infty}(\mathrm{R}) \rightarrow \mathrm{L}^{-\infty}(\mathrm{R} \Gamma)
$$

is equivalent to an inclusion of a direct summand of spectra.

Proof. We shall continue to discuss only the algebraic $K$-theory case, the other case being obvious modifications of these arguments. The reason the methods of [5] do not cover this case is that when $(\overline{E \Gamma}, \partial E \Gamma)$ is not metrizable, we do not know how to compute the continuously controlled $K$-theory of $(\overline{E \Gamma}, \partial E \Gamma)$. As far as Čech homology is concerned there are no such restrictions. Consider the following bornologies on $E \Gamma \times(0,1)$ 
1) The continuously controlled bornology of $(E \Gamma \times(0,1], E \Gamma \times 1)$

2) The pullback of the continuously controlled bornology on $([0,1], 0 \cup 1)$ via the projection map $E \Gamma \times(0,1) \rightarrow(0,1)$.

$3)$ The pullback of the continuously controlled bornology on $(\overline{E \Gamma}, \partial E \Gamma)$ via the projection map $E \Gamma \times(0,1) \rightarrow E \Gamma$.

We shall denote the intersection of all three bornologies by $\mathscr{B}(C \overline{E \Gamma})$. We denote the intersection of the last two mentioned bornologies by $\mathscr{B}(\Sigma \overline{E \Gamma})$. Finally we have the continuously controlled bornology $\mathscr{B}\left(C\left(E \Gamma_{+}\right), E \Gamma_{+}\right)$. Consider

$$
\mathscr{B}\left(C\left(E \Gamma_{+}\right), E \Gamma_{+}\right) \stackrel{a}{\leftarrow} \mathscr{B}(C \overline{E \Gamma}) \stackrel{b}{\rightarrow} \mathscr{B}(\Sigma \overline{E \Gamma}) .
$$

Without repeating the arguments of [5] it may help the understanding to think of these bornologies in terms of three different compactifications of $E \Gamma \times(0,1)$. The first compactification is $C \overline{E \Gamma}$, and the bornology $\mathscr{B}(C \overline{E \Gamma})$ expresses that we have continuous control at the bottom of the cone and at the cone point, and along $\partial E \Gamma \times(0,1)$ we only require control in the $\partial E \Gamma$ direction. The effect is that if $B$ is in this bornology and $((e, s),(f, t)) \in B$, then if $s$ is close to $0, t$ has to be close to $s$, if $e$ is close to a boundary point, then $f$ has to be close to $e$, and if $t$ is close to 1 , then $e$ has to be close to $f$ and $s$ must be close to $t$. The second compactification is induced by the first by collapsing $\overline{E \Gamma}$ to a point, so it is $\Sigma \overline{E \Gamma}$. This bornology ensures that the ends stay apart, we still have no restrictions in the suspension coordinate. It is easy to see that this bornology is a deloop of the continuously controlled bornology $\mathscr{B}(\overline{(} E \Gamma), \partial E \Gamma)$ in the sense that it is a deloop after applying $K^{-\infty}$. Finally the third bornology, the continuously controlled bornology $\mathscr{B}\left(C\left(E \Gamma_{+}\right), E \Gamma_{+}\right)$is obtained by collapsing $C(\partial E \Gamma)$. It was proved in [5, Corollary 2.10] that

$$
\Omega K^{-\infty}(E \Gamma \times(0,1), \mathscr{B}(C \overline{E \Gamma})) \Gamma \simeq B \Gamma_{+} \wedge K^{-\infty}(R)
$$

as spectra and [5, Lemma 2.3]

$$
\Omega K^{-\infty}(E \Gamma \times(0,1), \mathscr{B}(\Sigma \overline{E \Gamma}))^{\Gamma} \simeq K^{-\infty}(R \Gamma)
$$

and the map induced by $b$ is the assembly map [5, Section 3]. One element in the proof was to show that

$$
\left.\left.\mathscr{B}\left(E \Gamma \times(0,1), \mathscr{B}(C \overline{E \Gamma})^{\Gamma}\right)\right) \rightarrow \mathscr{B}\left(E \Gamma \times(0,1), \mathscr{B}\left(C\left(E \Gamma_{+}\right), E \Gamma_{+}\right)^{\Gamma}\right)\right)
$$

is an equivalence of categories. This is the point where it is used that the action is small at infinity. The fixed set consist of the equivariant maps, and the smallness then ensures that the control conditions are automatically satisfied along $\partial E \Gamma \times[0,1]$. 
Lemma 12.2. The pullback maps

$$
\check{h}\left(\Sigma \partial E \Gamma, K^{-\infty}\right) \rightarrow \check{h}\left(\Sigma \partial E \Gamma, \mathscr{B}(\Sigma \overline{E \Gamma}) ; K^{-\infty}\right)
$$

and

$$
\check{h}\left(C(\partial E \Gamma) \cup \overline{E \Gamma} ; K^{-\infty}\right) \rightarrow \check{h}\left(C(\partial E \Gamma) \cup \overline{E \Gamma}, \mathscr{B}(C \overline{E \Gamma}) ; K^{-\infty}\right)
$$

are homotopy equivalences of spectra

Proof. The bornologies $\mathscr{B}(C \overline{E \Gamma})$ and $\mathscr{B}(\Sigma \overline{E \Gamma})$ are not small at infinity when we compactify $E \Gamma \times(0,1)$ by $C \partial E \Gamma \cup \overline{E \Gamma} \times 1$ and $\Sigma(\partial E \Gamma)$ respectively. An open subset $U$ is bornologically saturated if and only if $(x, t) \in \partial E \Gamma \times(0,1)$ belongs to $U$ implies $x \times(0,1) \subset U$. The smallest bornologically saturated open set containing the cone point will thus be $C \partial E \Gamma-\overline{E \Gamma}$. The smallest bornologically saturated open set containing a suspension point is the whole space minus the other suspension point. Except for the trivial covering where one of the open sets is the whole space we thus get that the nerve of a bornologically saturated open covering of $\Sigma \partial E \Gamma$ is precisely the suspension of the nerve of a covering $\partial E \Gamma$

Consider the diagram where we denote $E \Gamma \times(0,1)$ by $X$ and omit the ring $R$ from the notation.

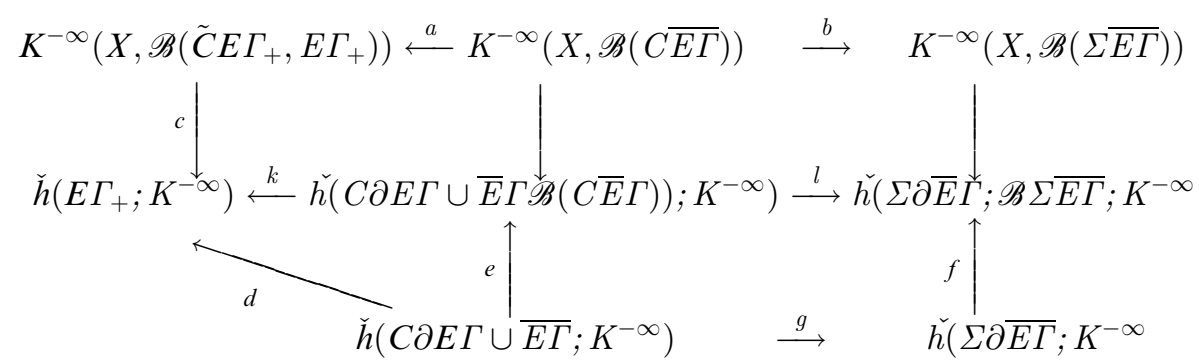

All the maps are equivariant maps. The map $b^{\Gamma}$ on fixed sets is the assembly map , and the map $a^{\Gamma}$ is a homotopy equivalence as discussed above. By definition $K^{-\infty}\left(X, \mathscr{B}\left(\tilde{C} E \Gamma_{+}, E \Gamma_{+}\right)\right)$is the same as $K^{-\infty}\left(\tilde{C} E \Gamma_{+}, E \Gamma_{+}\right)$, and we proved in [5, Theorem 2.11] that

$$
K^{-\infty}\left(\tilde{C} E \Gamma_{+}, E \Gamma_{+}\right)^{\Gamma} \rightarrow K^{-\infty}\left(\tilde{C} E \Gamma_{+}, E \Gamma_{+}\right)^{h \Gamma},
$$

the map from the fixed set to the homotopy fixed set is a homotopy equivalence. Since equivariant maps that are homotopy equivalences induce homotopy equivalences on homotopy fixed sets we will be finished once we prove that $c, k$ and $l$ are homotopy equivalences. The map $c$ is a homotopy equivalence since $E \Gamma_{+}$is metrizable by Theorem 10.16. We have argued above that $e$ and $f$ are homotopy equivalences. The maps $d$ and $g$ are in- 
duced by collapsing a contractible subset so by excision and homotopy invariance they induce homotopy equivalence and we are done.

Finally we state a more useful theorem where the proof is a slight variation of the proof above.

Theorem 12.3. Assume $\Gamma$ is a group with a finite $B \Gamma$ and that $E \Gamma$ has an equivariant compact Hausdorff compactification which is Čch contractible and such that there exists a family of coverings $\mathscr{F}$ of $\partial E \Gamma$ by sets which are saturated with respect to the bornology $\mathscr{B}^{\Gamma}$ on $E \Gamma$ (e. g. boundedly saturated) which is invariant under the group action and satisfies $\breve{h}(\partial E \Gamma ; T) \rightarrow \check{h}(\partial E \Gamma, \mathscr{F} ; T)$ is a homotopy equivalence, where $T$ is the relevant spectrum $K^{-\infty}(R)$ or $\mathrm{L}^{-\infty}(\mathrm{R})$. Then

a) If $R$ is any ring then the assembly map

$$
B \Gamma_{+} \wedge K^{-\infty}(R) \rightarrow K^{-\infty}(R \Gamma)
$$

is equivalent to an inclusion of a direct summand of spectra.

b) If $R$ is a ring with involution such that $K_{-i}(R)=0$ for $i$ sufficiently large then the assembly map

$$
B \Gamma_{+} \wedge \mathrm{L}^{-\infty}(\mathrm{R}) \rightarrow \mathrm{L}^{-\infty}(\mathrm{R} \Gamma)
$$

is equivalent to an inclusion of a direct summand of spectra.

Proof. The proof is a slight variation of the proof above using the bornology on $E \Gamma$ induced by the given collection of subsets of $\partial E \Gamma$ occurring in the family $\mathscr{F}$ instead of the continuously controlled bornology in point (2) above. Our condition ensure that equivariant maps are automatically controlled with respect to this bornology even though the action may not be small at infinity.

REMARK 12.4. The theorem above may be generalized in various ways. We do not necessarily have to work with open sets. As long as the coverings satisfy that any pair of intersections is an excisive pair, these kind of methods can be used. We shall not try to formulate the most general theorem that can be formulated along these lines.

\section{REFERENCES}

1 J.F. Adams, Stable homotopy and generalized homology, Chicago Lectures in Mathematics, University of Chicago Press, 1974.

2 D. R. Anderson, F. Connolly, S. C. Ferry, and E. K. Pedersen, Algebraic K-theory with continuous control at infinity., J. Pure Appl. Algebra. 94 (1994), 25-47. 
3 A. K. Bousfield and D. M. Kan, Homotopy, Limits, Completions, and Localizations, Lecture Notes in Math. 304, 1972.

4 G. Carlsson, Bounded K-theory and the assembly map in algebraic K-theory, Proceedings 1993 Oberwolfach Conference on the Novikov Conjectures, Rigidity and Index Theorems Vol. 2 (S. C. Ferry, J. Rosenberg, and A. A. Ranicki, eds.), London Lecture Note Ser. 227, 1995, pp. 5-127.

5 G. Carlsson and E. K. Pedersen, Controlled algebra and the Novikov conjectures for K- and L-theory, Topology 34 (1995), 731-758.

6 G. Carlsson, E. K. Pedersen, and J. Roe, Controlled $C^{*}$-algebra theory and the injectivity of the Baum-Connes map, Preprint, 1994.

7 G. Carlsson, E. K. Pedersen, and W. Vogell, Continuously controlled algebraic K-theory of spaces and the Novikov conjecture, Math. Ann. (To appear).

8 D. A. Edwards and H. M. Hastings, $C^{4} e c h$ and Steenrod Homotopy Theories with Applications to Geometric Topology, Lecture Notes in Math. 542, 1976.

9 E. M. Friedlander, Etale Homotopy of Simplicial Schemes, Ann of Math Stud. 1982.

10 B. Goldfarb, Novikov conjectures for arithmetic groups withs with large actions at infinity, Ph.D. thesis, Cornell University, 1995.

11 M. Gromov, Hyperbolic groups, Math. Sci. Res. Inst. Publ. 8 (1987), 75-264.

12 N. Higson, E. K. Pedersen, and J. Roe, $C^{*}$-algebras and controlled Topology, K-theory 11 (1997), 209-239.

13 D. S. Kahn, J. Kaminker, and C. Schochet, Generalized homology theories on compact metric spaces, Michigan. Math. J. 24 (1977), 203-224.

14 J. Milnor, On the Steenrod homology theory, Proceedings 1993 Oberwolfach Conference on the Novikov Conjectures, Rigidity and Index Theorems Vol. 2 (S. C. Ferry, J. Rosenberg, and A. A. Ranicki, eds.), London Math. Soc. Lecture Note 226, 1995, pp. 79-96.

15 E. K. Pedersen and C. Weibel, K-theory homology of spaces, Algebraic Topology, Proceedings Arcata 1986 (Berlin-New York) (G. Carlsson, R. L. Cohen, H. R. Miller, and D. C. Ravenel, eds.), Lecture Notes in Math. (1989), 346-361.

16 D. G. Quillen, Higher algebraic K-theory, Proceedings of Battelle Seattle Algebraic $K$-theory Conference 1972 (Berlin-New York), Lecture Notes in Math. 341 (1973), 85-147.

17 R. W. Thomason, Homotopy colimits in the category of small categories, Math. Proc. Camb. Phil. Soc. 85 (1979), 91-109.

18 R. W. Thomason, First quadrant spectral sequences in algebraic $K$-theory via homotopy colimits, Comm. Alg. 10 (1982), 1589-1668.

19 M. Weiss and B. Williams, Assembly, Proceedings 1993 Oberwolfach Conference on the Novikov Conjectures, Rigidity and Index Theorems Vol. 2 (S. C. Ferry, J. Rosenberg, and A. A. Ranicki, eds.), London Math. Soc. Lecture Note 227, (1995), 332-352.

DEPT. OF MATH.

STANFORD UNIVERSITY USA
DEPT. OF MATH

BINGHAMTON UNIVERSITY

USA 